\title{
Initial Evidence of Research Quality of Registered Reports Compared to the Standard
}

\section{Publishing Model}

Courtney K. Soderberg ${ }^{1 \#}$, Timothy M. Errington ${ }^{1 \#}$, Sarah R. Schiavone ${ }^{2}$, Julia Bottesini ${ }^{2}$, Felix Singleton Thorn ${ }^{3}$, Simine Vazire ${ }^{2,3}$, Kevin M. Esterling ${ }^{4}$, and Brian A. Nosek ${ }^{1,5^{*}}$

${ }^{1}$ Center for Open Science, Charlottesville, Virginia, USA

${ }^{2}$ Department of Psychology, University of California, Davis, Davis, California, USA

${ }^{3}$ School of Psychological Sciences, University of Melbourne, Melbourne, Victoria, Australia

${ }^{4}$ Department of Political Science, University of California, Riverside, Riverside, California, USA

${ }^{5}$ Department of Psychology, University of Virginia, Charlottesville, Virginia, USA

\# Contributed equally.

* Correspondence to Brian A. Nosek: nosek@cos.io

Keywords: Registered Reports, research quality, meta-research, peer review, publishing models 


\begin{abstract}
In Registered Reports (RRs), initial peer review and in-principle acceptance occurs before knowing the research outcomes. This combats publication bias and distinguishes planned and unplanned research. How RRs could improve the credibility of research findings is straightforward, but there is little empirical evidence. Also, there could be unintended costs such as reducing novelty. 353 researchers peer reviewed a pair of papers from 29 published RRs from psychology and neuroscience and 57 non-RR comparison papers. RRs outperformed comparison papers on all 19 criteria (mean difference $=0.46$; Scale range -4 to +4 ) with effects ranging from little improvement in novelty $(0.13,95 \%$ credible interval $[-0.24,0.49])$ and creativity $(0.22,[-0.14,0.58])$ to larger improvements in rigor of methodology $(0.99,[0.62,1.35])$ and analysis $(0.97,[0.60,1.34])$ and overall paper quality $(0.66,[0.30,1.02])$. RRs could improve research quality while reducing publication bias and ultimately improve the credibility of the published literature.
\end{abstract}

Word count $=149$ 
Publication of journal articles is the primary mechanism for communicating the results of empirical research and a key incentive for researchers to advance in their careers. The standard model of peer review and publication is vulnerable to biases that can reduce the credibility of the published literature ${ }^{1-5}$. Some kinds of results are more likely to be published than others.

Positive results -- finding that a treatment is effective or that there is a relationship between two variables -- are more likely to be published than negative results ${ }^{6-12}$. Novel or innovative results are more likely to be published than replications or incremental extensions of existing findings ${ }^{13-15}$. And, clean papers in which the evidence consistently supports the proposed conclusion are more likely to be published than papers with equivocal evidence, exceptions, or other uncertainties ${ }^{16,17}$. Attention paid to positive, novel, and clean findings could come at the expense of evaluating the quality and rigor of the research for making publication decisions. This may contribute to publication bias, selective reporting, and questionable research practices that reduce the credibility of the published literature ${ }^{18-25}$.

Registered Reports (RRs) are designed to address these biases and improve the quality of research ${ }^{1-3}$. With RRs, the first stage of peer review occurs prior to knowing the research outcomes of the key study or studies, in most cases before data collection has occurred. Reviewers evaluate the importance of the research question and the quality of the methodology used to evaluate that question. The findings cannot distract reviewers from evaluating research quality and rigor because there are no findings yet. Expert review in advance should facilitate improving the methodology rather than just pointing out limitations of findings already obtained. If accepted, the authors receive an in-principle commitment from the journal to publish the results as long as the research is performed as proposed with evidence of competent execution and interpretation. Decisions to publish are therefore based on the perceived importance of the 
research question and the quality of the methodology, not whether the findings are positive, novel, and clean.

More than 250 journals have adopted RRs since 2013 on the theorized promise of improving rigor and credibility. Initial evidence suggests that RRs are (1) effective at mitigating publication bias with a sharp increase in publishing negative results compared to the standard model $^{26,27}$, and (2) cited as often or even more than other articles in the same journals ${ }^{28}$. However, there is no evidence about whether scholars perceive RRs to have higher, lower, or similar research quality compared with papers published in the standard model. The RR format could also have costs such as authors pursuing less interesting questions or conducting less novel or creative research 29,30 .

We conducted an observational investigation of perceptions of the quality and importance of RRs compared to the standard model across a variety of outcome criteria. We recruited 353 researchers to each peer review a pair of papers, one from 29 RRs from psychology and neuroscience and one from 57 matched non-RR comparison papers. Comparison papers addressed similar topics, about half were by the same first or corresponding authors and about half were published in the same journal. RRs is a popular format for replication studies ${ }^{3,31}$, but replications are rare in the standard model so we excluded replication RRs. Researchers were assigned to papers according to their self-reported expertise based on the papers' keywords. Researchers self-reported that they were qualified to review the papers on average ( $N=353$; RR $M=3.74, S D=1.02$; Comparison paper $M=3.59, S D=1.07$; Range 1 [not at all qualified] to 5 [substantially qualified]). Reviewers evaluated 19 outcome criteria including quality, rigor, novelty, creativity, and importance of the methodology and outcomes of the papers. In some RRs, authors submitted preliminary studies as initial evidence supporting the approach of the proposed last study that was peer reviewed before the findings were known. If 
the RR or non-RR had multiple studies (27 of 86 ), then evaluation of methods and results were about the last study only. We also coded the papers on objective qualities of the research such as sample size, preregistration, and data sharing. The findings provide an initial empirical test of the quality of research published using RRs compared with the standard model.

\section{Results}

Preregistered primary outcomes. Reviewers read and evaluated each paper in three steps. They first read and evaluated the introduction, initial studies (if any), and methodology of the last study before knowing the last study's results. Next, they read and evaluated the last study's results and the discussion. Finally, they read the paper's abstract and provided final assessments of the paper. We preregistered analysis plans for comparing the difference between ratings of RRs and control articles on each criterion using a within-subjects comparison to maximize power and a between-subjects comparison for the article that reviewers were randomly assigned to read first. As estimated effects were similar across analysis strategies, we emphasize reporting the more precise within-subjects estimates. Our preregistered within-subjects model included a separate model for each outcome, but upon seeing the correlational structure of the outcomes (Extended Data Figure 1), we decided to use a multi-level model including all outcomes with partial-pooling to mitigate multiple comparisons concerns.

The results of that model are presented in Figure 1 and are consistent with the results of the preregistered within-subjects model. In the figure and paragraph below, we report point estimates and $95 \%$ credible intervals on the raw response scales. In text, we also report a standardized effect size metric and . Credible intervals do not have the same interpretation as confidence intervals that are commonly reported in null hypothesis significance testing. Given the evidence provided by the observed data and weakly informative priors, there is a $95 \%$ 
posterior probability that the true estimate lies within the credible interval. This is distinct from common uses of confidence intervals in null hypothesis significance testing to declare a result 'statistically significant' based on whether the interval includes 0 .

Before learning the findings of the last study, compared to control articles, reviewers evaluated the RRs' last study as having a more rigorous methodology (estimate $=0.99,95 \%$ Credible Interval $[0.62,1.35]$, standardized ES $=0.43,95 \%$ Credible Interval $=[0.27,0.58]$ ), higher quality methodology (estimate $=0.77,[0.40,1.12]$, standardized $E S=0.32,[0.17,0.47]$ ), higher estimates of what will be learned (estimate $=0.22,[0.05,0.38]$, standardized $E S=0.17$, $[0.01,0.32])$, better alignment between the research question and methodology (estimate $=$ $0.37,[0.01,0.73]$, standardized $E S=0.19,[0.01,0.37])$, higher quality research question (estimate $=0.39,[0.03,0.76]$, standardized ES $=0.20,[0.02,0.38])$, more important research regardless of what results will be observed (estimate $=0.27,[-0.09,0.63]$, standardized $\mathrm{ES}=$ $0.12,[-0.04,0.28])$, more creative methodology (estimate $=0.22,[-0.14,0.58]$, standardized ES $=0.11,[-0.07,0.28]$ ), and more novel research questions (estimate $=0.13,[-0.24,0.49]$, standardized $E S=0.06,[-0.12,0.25])$.

After reading the results and discussion of the last study, compared to control articles, reviewers evaluated the RRs as having a more rigorous analysis strategy (estimate $=0.96$, $[0.60,1.34]$, standardized ES $=0.46,[0.28,0.63])$, more justified conclusions based on the results (estimate $=0.65,[0.29,1.01]$, standardized $E S=0.31,[0.14,0.48])$, higher quality of the results (estimate $=0.61,[0.25,0.97]$, standardized $E S=0.29,[0.12,0.47])$, higher quality of the discussion (estimate $=0.40,[0.04,0.77]$, standardized $E S=0.19,[0.02,0.37]$ ), higher estimates of what was learned (estimate $=0.40,[0.03,0.77]$, standardized $E S=0.17,[0.01,0.32]$, more innovative results (estimate $=0.29,[-0.08,0.65]$, standardized $E S=0.13,[-0.04,0.30]$, and more important findings (estimate $=0.28,[-0.08,0.64]$, standardized $E S=0.12,[-0.04,0.28]$ ) 
After finishing the paper, compared to control articles, reviewers evaluated the RRs as having higher overall quality (estimate $=0.66,[0.30,1.02]$, standardized $\mathrm{ES}=0.32,[0.15$, 0.50]), producing more important discoveries (estimate $=0.38,[0.02,0.75]$, standardized $\mathrm{ES}=$ $0.17,[0.01,0.34]$ ), better alignment between abstract and findings (estimate $=0.33,[-0.03$, 0.69], standardized ES $=0.21,[-0.02,0.44]$ ), and more likely to inspire new research (estimate $=0.18,[-0.19,0.54]$, standardized $E S=0.08,[-0.08,0.24])$.

In the aggregate, RRs outperformed comparison articles on all 19 criteria with an average magnitude of 0.46 and a range of 0.13 to 0.96 . Twelve of the 19 criteria showed performance advantages with 95\% credible intervals not including zero.

We repeated the analysis as a between-subjects comparison using only the randomly assigned first reviewed article. This substantially reduces precision compared to the within-subjects design, but has the advantage of a possibly more "pure" assessment of articles before reviewers might have noticed and compared differences between articles in the within-subjects design, potentially exaggerating observed effects. As with the within-subjects analysis, our originally preregistered model included a separate model for each outcome, but these results were similar to a model that included all outcomes (see Supplementary Information). The observed effects from the between subject model with all outcomes showed a similar pattern with an average effect size of 0.43 and a range of 0.04 to 0.93 (Supplementary Table 1).

Characteristics of the papers and reviewers. When preparing the papers for review, we removed explicit mention of RRs in the text, but we did not otherwise attempt to blind reviewers from information about the methodology because their task was to evaluate the methodology. After completing the reviews, we informed reviewers about the study's focus on RRs and preregistration and asked about their experience and opinions. Less than half (47\%; 
154/326) had ever preregistered a study, and 14\% (41/299) had submitted a RR. Even so, reviewers reported being relatively familiar with preregistration $(M=3.90 ; S D=1.22$; Range 1 [not at all] to 5 [substantially] $)$ and $\mathrm{RRs}(M=3.24, \mathrm{SD}=1.33)$. Reviewers also reported believing that RRs can improve research rigor $(M=2.01, S D=1.32$; Range -4 [substantially worsen] to 4 [substantially improve]) and quality $(M=1.71, S D=1.53)$. Finally, a majority of reviewers correctly identified the RR as a RR (54\%; 190 correct, 38 incorrect, 125 unsure) and the comparison paper as not a RR (54\%; 192 correct, 30 incorrect, 131 unsure).

\section{Qualification of outcomes by characteristics of the papers and reviewers. We} conducted exploratory analyses of how characteristics and beliefs of the reviewers might qualify the performance outcomes for RRs compared with other papers. Our sample was positive towards RRs and familiar with the format with some variation, so we explored how that may have affected our results. Beliefs about quality and rigor of RRs were highly correlated $(r=0.82)$, as were familiarity with $\mathrm{RR}$ and preregistration $(r=0.74)$, so we averaged these questions to form single familiarity and improvement belief items. To allow for a non-linear relationship, we kept the resulting averages as categorical variables rounding half values to create a 5 and 6 level factor, respectively. These were then included as random effects in two within-subjects models to allow for partial pooling across factor levels and DVs to mitigate multiple comparison concerns $^{32}$. Those reporting the highest $(M=0.54,95 \%$ credible interval $[0.12,0.99])$ and next highest level of familiarity $(0.45,[0.06,0.83])$ perceived a larger performance advantage for RRs than those reporting the lowest level of familiarity $(0.32,[-0.24,0.77])$ but similarly with the second lowest level of familiarity $(0.49,[0.06,0.95])$ and the middle level of familiarity $(0.42$, [0.02, 0.81]; See Extended Data Figure 2 and Supplementary Table 2 for more details). And, unsurprisingly, those reporting very strong $(0.82,[0.26,1.40])$, strong $(0.52,[0.11,0.94])$, and moderately strong beliefs that RRs improve rigor $(0.46,[0.03,0.89])$ showed a larger 
performance advantage for RRs those who were slightly favorable toward RRs $(0.26,[-0.20$, $0.71])$, neutral toward RRs $(0.45,[-0.07,0.97])$ or those who believed RRs actually decreased quality and rigor $(0.24,[-0.45,0.82]$; See Extended Data Figure 3 and Supplementary Table 3 for more details). It is not surprising that believing that the RR format improves rigor and quality would translate to higher ratings of rigor and quality of rating individual RRs, just as it would not be surprising that believing that random assignment, random sampling, and blinding improves quality would translate to higher ratings when those features were present versus absent. Notably, however, every subgroup estimate was in the direction of a performance advantage for RRs, even among those reviewers who believed that RRs were neutral or negative for enhancing rigor and quality in general.

We also explored whether the performance advantages for RRs were present only for those that had correctly identified articles as RRs or not. To the extent that certain characteristics of articles (e.g., reporting style or content) are viewed as more rigorous and also are a good indicator of an article being a $R R$, it would not be surprising to see a relationship between the RR performance advantage and correctly guessing each type of article. A more interesting question is whether even reviewers that did not correctly guess article type would still perceive improved quality and rigor. In the within-subjects model, 19 of 19 outcome criteria remained directionally consistent with an RR performance advantage for reviewers that guessed one paper correctly ( $n=168)$, and 14 of 19 outcome criteria remained directionally consistent for reviewers that guessed neither paper correctly $(n=87)$. And, for both, the average raw effect sizes were weaker than observed in the aggregate data (one paper correctly guessed: $M=0.44$, Range $=-0.23-0.71$; neither paper correctly guessed: $M=0.10$, Range $=-0.03-0.35$; See Extended Data Figure 4 and Supplementary Table 4 for more details). Perceived performance advantages of RRs are associated with noticing that the papers are RRs, but these results 
provide modest evidence that reviewers may perceive some performance advantages even if they do not notice or correctly guess that a paper is a RR.

Coding outcomes. We coded objective features of the articles that could be associated with greater rigor and transparency. Here, the unit of analysis is the article, substantially reducing precision because of the limited sample size. Table 1 summarizes descriptive evidence that statistical reporting errors as identified by statcheck ${ }^{33}$ occurred infrequently. Preregistration occurred much more frequently with RRs, as should be expected because the format includes precommitment to design and planned analysis. Lack of preregistration means that a link to a publicly available precommitted design and analysis plan was not included in the paper for verifiability. Observing less than $100 \%$ preregistration indicates an opportunity for improving formal documentation of $\mathrm{RRs}^{34,35}$. Sharing materials and data were observed more frequently in RRs than comparison articles. Finally, median sample sizes were notably larger in RRs and sample size justifications and online data collection were more common, but studies with difficult to reach populations were less common.

\section{Discussion}

Reviewers rated RRs similarly or more positively as non-RR comparison articles across 19 outcome criteria (mean performance advantage $=0.46$, range $=[0.13,0.96])$. This advantage held whether considering a within-subjects design or a between-subjects design comparing whichever article reviewers examined first. It also held whether reviewers had experience or familiarity with RRs or preregistration, and was weaker but not reversed if they held equivocal beliefs about RRs in general. The performance advantage held among criteria that were assessed before showing the findings $(M=0.45$; Range $=0.13-0.97)$, after showing the findings $(M=0.39 ;$ Range $=0.18-0.66)$, and after finishing the paper $(M=0.51$; Range $=0.28-0.97)$. Finally, RRs investigated in this study were associated with larger median sample sizes, more 
sharing of data and materials, stronger presence of preregistration than comparison articles, and somewhat less use of hard to reach samples.

RRs' performance advantage was strongest on the factors that should be most directly the basis of peer review: methodology and analysis rigor and quality. The RR performance advantage extended to characteristics of design such as alignment between the question and methodology and reporting such as alignment between the findings and interpretation. Skeptics have suggested that the planning required for RRs could undermine discovery, reduce novelty and creativity, promote boring research, and undermine innovation ${ }^{29,30}$. We found no support for these speculations. In fact, for none of the 19 outcome criteria did the standard model outperform RRs.

Limitations and Constraints on Generality. This comparative study provides important initial evidence about the quality and performance of RRs for research credibility. However, it is not a definitive comparative investigation of RRs versus the standard publishing model. The naturalistic design of this study means that there was no random assignment of research to be conducted as an RR or not. The naturalistic design also means that the included RRs were not performed by a random subset of authors or at a random subset of journals, as authors and editors self-select whether to use RRs. The matching strategy we used was designed to reduce these concerns, minimising confounding explanations by matching on topic as well as on journal and lead author. A randomized trial of RRs would be worth conducting, although it will be difficult to avoid researchers becoming aware of their participation and treatment group. Our naturalistic approach is arguably less subject to reactivity on the part of reviewers because the review is naturalistic and retrospective. Also limiting the generalisability of these results is the simple fact that the RR publication format is new. Behavior during early adoption of publishing practices may not be the same as later adoption. Early adoption could suffer from initial weaknesses in 
RR implementation, or it could be particularly high quality due to extra attention based on its novelty. Simultaneously, the researchers and editors who are early adopters of the format may be different in important ways than later adopters. Another limitation is that we used peer review evaluation of research quality rather than other assessments of research quality, such as assessing whether Registered Reports produce findings that are more replicable or generalizable. While such outcomes are difficult or resource-intensive to assess, they are necessary in combination with expert evaluation to get a complete understanding of research quality. Finally, we recruited reviewers and matched them to topics based on expertise similarly to standard peer review. However, we cannot estimate their representativeness of reviewers in general. Despite these limitations, this study provides a basis of evidence to expand the use of RRs in research, and should spur follow-up research to examine the generality of these findings. We do not have strong reasons to expect that these findings will fail to generalize to other fields, other methodologies, and other reviewers. Nevertheless, these all deserve empirical scrutiny.

Conclusion. Registered Reports were conceived to alter the incentives for authors and journals away from producing novel, positive, clean findings and toward conducting and publishing rigorous research on important questions ${ }^{1-3}$. Making acceptance decisions prior to knowing the outcomes should and does mitigate publication bias favoring positive results ${ }^{26,27}$. The present evidence indicates that Registered Reports are associated with reviewers perceiving greater rigor and quality without costs on research importance or creativity compared with the standard model. We speculate that the higher quality of RRs is a function of three factors: authors put greater emphasis on defining their research questions and designing their methodologies to achieve acceptance, peer reviewers and editors are more likely to evaluate 
the methodology when there aren't findings to distract or excuse poor methodological decisions, and peer review can prompt study design improvements before conducting the research.

Shifting the incentives toward quality and rigor may have a variety of salutary effects on the published literature and research culture. The pressure to achieve positive results creates corrosive incentives that reward questionable research practices and undermine the credibility of findings ${ }^{4,36,37}$. Realigning reward systems with the values of quality and rigor could improve research credibility and accelerate the discovery of knowledge, solutions, and cures. Registered Reports are not a panacea, but the evidence suggests that they offer clear and tangible benefits to improving research and the research culture.

\section{Methods}

This research protocol was reviewed and approved by the University of Virginia IRB for Social and Behavioral Sciences (IRB-SBS; Protocol \#3077). All participants consented to participate.

Registered Report Article Selection. The study was conducted using RRs published from January 1, 2014 to April 18, 2018 that were identified from the 102 journals that offered RRs at that time either as a regular submission option or as part of a single special issue (https://cos.io/rr). An ongoing list of all published RRs are available in this database (https://bit.ly/2pJRYz3). For this study, we used RRs from psychology and neuroscience. We set these inclusion criteria for efficient implementation of the comparison matching process, for feasibility of defining a coding scheme that could be administered consistently across studies, and for feasibility of recruiting peer reviewers. We also excluded RRs reporting replications. Replications are rare in the non-RR literature and popular with the RR format. Including replications in our RR sample would induce a confound as we would not be able to find enough matched non-RR replication studies. Supplementary Figure 1 shows the effect of our inclusion 
and exclusion criteria on our article sample. In total, we included 29 RRs reporting novel research from psychology and neuroscience (Supplementary Table 5).

Comparison Article Selection. We used existing RRs that investigated novel research questions and a sample of comparison articles from the published literature. This has the advantage of ecological validity, but the disadvantage of being a quasi-experimental design that could introduce threats to construct validity of RRs versus the standard model. We preregistered two complementary, bias-minimizing matching procedures to select comparison articles for each RR. One procedure matched articles primarily based on journal and date of publication to emphasize comparability on publishing selectivity, selection criteria, and journal style and standards. The other procedure matched articles primarily based on lead authors and date of publication to emphasize comparability of research style of those reporting the research. Each of the 29 RRs, with one exception, had matched articles for both selection criteria resulting in 57 comparison articles (see Supplementary Information for details), for a total of 86 articles (Supplementary Table 5). This systematic matching process minimizes selection bias and maximizes opportunity to observe variations in qualities of articles that are plausibly attributable to whether the article was an RR or not.

Article Coding. We coded the last study of each article on objective criteria including sample size and sample justifications, whether participants were recruited from difficult to reach populations, and study design (experimental or non-experimental). Coders also identified what they considered to be the study's primary outcome (see Supplementary Information for details). Two individual raters were randomly assigned to each article to independently apply the coding scheme for assessing objective characteristics of methodological rigor and reporting of outcomes. Agreement was computed using Krippendorff's alpha reliability coefficient with the “irr" package in R. Agreement was 0.943 for sample size, 0.788 for sample justification, 0.782 
for difficult to reach population, and 0.576 for study design. In cases of disagreement, a third coder resolved any coding disagreements. We also coded whether articles reported studies that were preregistered, had open materials or open data, or collected data online. To do so, we scraped and analyzed text from each article to identify terms referencing each variable, which were confirmed by an individual coder. We did not plan to conduct inferential statistics for the coding. Instead, we planned to report counts, means, and standard deviations for coded variables across $\mathrm{RR}$ and comparison articles.

We also ran each paper through the $\mathrm{R}$ package statcheck ${ }^{33,38}$ to identify errors in statistical tests reported in APA-style. Errors were those in which the reported statistical estimate, $p$-value, and degrees of freedom contained an inconsistency. Decision errors were those in which the statistical estimate was reported as statistically significant but the recomputed value was not, or vice versa.

Peer Reviewer Sample. We planned to obtain a sample size of 360 survey respondents (average of $\sim 12$ respondents per RR). This is equivalent to achieving $96 \%$ power to detect a medium effect size (Cohen's $d$ of 0.4 ), assuming the between-article pair proportion of variance is similar to the between-lab proportion of variance observed in multi-lab studies (3.85\%), or $80 \%$ power to detect the same effect size assuming a higher between-article proportion of variance ( 10\%; script: https://osf.io/fgkrs/). With our analysis strategy, we did not need to achieve perfect matching of sample size for each RR; however, we aimed for an even distribution of respondents across RRs while prioritizing maximizing expertise alignment. In Supplementary Table 6 , we report the number of respondents per RR article pair.

We recruited reviewers with individual emails starting October 16, 2019 and ending December 23, 2019. The recruiting email invited them to participate in a study to peer review two published articles and evaluate qualities such as creativity and rigor on rating scales, a task 
that would take up to 2 hours for compensation of $\$ 80$. Potential reviewers were given a 3-week window from the date of invitation to complete the reviews. And, finally, to facilitate non-experts self-selecting out of participating, the recruiting email identified eligibility as researchers with expertise in the subdisciplines of the selected articles.

Our primary sample was a list of emails of 7,100 faculty in U.S.-based psychology departments that had been constructed in 2017. With the passage of time, some of the email addresses were no longer valid due to faculty retirement or relocation. Also, a majority were in subdisciplines not represented in our sample of RRs making them unlikely to meet eligibility criteria. Nevertheless, this sample of email addresses provided an unbiased approach to recruiting potential peer reviewers. To achieve our target sample size and maximize coverage of peer reviews across the selection of articles, we supplemented this primary recruiting strategy with additional directed recruiting of reviewers representing specific topical areas. We identified 31 journals in Web of Science that clearly published research related to the topics of our sample of papers. We then extracted 398 European email addresses and 337 U.S. email addresses of corresponding authors from Web of Science from recent publications in those journals.

A total of 7,835 email invitations were sent and 353 peer reviewers completed at least one outcome measure $(4.5 \%)$. We do not know the breakdown of reasons for declining to participate, but almost all of the more than 100 spontaneous "decline to participate" responses indicated that the specific disciplinary topics were outside their expertise, they did not have time to do the reviews in the next 3 weeks, or they had retired and were no longer reviewing. Participant reported gender was $56 \%$ male, $42 \%$ female, and $2 \%$ preferred not to say. We did not assess participant age.

Prior to consenting for the study, potential reviewers completed a short eligibility assessment to determine whether their areas of expertise matched any of the articles in the 
sample. Potential reviewers first identified the subdiscipline closest to their area of expertise (social-personality psychology, cognitive psychology, neuroscience, or none of the above). If they selected "none of the above" they were informed that they did not match any articles and thanked for their interest in participating. If they selected social-personality psychology they were presented with five options (media/applied psychology, group processes and intergroup relations, positive psychology, personality psychology, or attitudes and social cognition). If they selected a disciplinary area matching our set of articles, they were presented with article keywords in sets and identified the set of keywords that most closely matched their expertise. The article keyword sets were constructed by combining the keywords from an RR and its two matched comparison articles. This approach enabled us to match researchers based on expertise without revealing the selected articles and without biasing selection by expertise on the RR or comparison articles.

After selecting a keyword set, potential reviewers consented to participate and then were assigned to review the selected $\mathrm{RR}$ and a randomly selected comparison article from the pair. The two articles were presented in a randomized order via Qualtrics and reviewers completed one review before proceeding to the next article. Reviewers could pause and return to complete the reviews if they could not complete the task in a single sitting. 353 reviewers were matched to articles and completed at least one outcome measure. Because we matched on expertise, the number of reviewers was not equally distributed across articles. Of the $29 \mathrm{RR}$ and control article matches, each had an average of 12.5 reviewers with a low of 3 and a high of 24 (SD $=5.59)$.

Structured Peer Review Survey. We developed a structured peer review survey to evaluate the papers on 19 qualities. Each reviewer completed a subjective evaluation of two papers -- an RR and a control paper randomly selected from the RR's two comparison papers. 
Papers were lightly redacted to remove any incidence or indication of the words "Registered Report." We expected that at least some papers and some reviewers would still be able to identify RRs. We considered trying to completely blind the studies. However, in test cases we found that this resulted in removal of content that was directly relevant for evaluating rigor and transparency of the study design and findings. At the end of the study, we asked reviewers whether each of the papers was published as an RR. We also assessed reviewers' familiarity with and beliefs about RRs in general.

To minimize the influence of the findings on evaluation of the methods, the content of each paper was presented and reviewed in the following fixed order: 1) Introduction, initial studies, and methods of the last study, 2) results of the last study and discussion, 3) abstract. Section headings, text, tables, figures, and references were included, while other paper attributes (e.g. Author(s), Journal) were not included. Reviewers completed all measures for one section before proceeding to the next section.

Reviewers provided quantitative responses on bipolar 9-point Likert scales ranging from -4 (substantially less than the average study) to 4 (Substantially more than the average study) with 0 indicating equivalence to "the average study [article]." The average study reference point is important for encouraging full use of the scale rather than making an absolute assessment of "meets publishing criterion" as is typical for peer review decisions to accept or reject papers for publication. Each section included a short open-response box if reviewers wished to explain their responses. These responses were retained for exploratory purposes. All survey questions focused on the last study of the paper unless specifically stated to comment on the paper as a whole.

Preregistered Analyses. We were interested in the causal effect of an article being an RR on our survey questions. Our data are nested: Reviewers are nested within article pairs, 
and article pairs are nested within discipline. For each article pair, there is an effect of article type on each survey response. The overall effect of interest is the average effect of article type across article pairs. This is similar to a meta-analytic effect, with the article pairs being similar to effects from individual studies. We used multilevel modeling to account for our nesting and to allow the effect of article type to vary across the different article pairs, partially pooling the estimates to create an overall average effect. In our preregistration, responses to the survey questions were modeled separately, running a mixed-effects model for each outcome, as we did not have strong a priori predictions about higher order latent constructs and so were not sure if the assumption that would be required to run all outcomes in a single model would hold. Data analysis was not performed blind to the conditions.

\section{Within vs. Between Effects}

For each article pair, there were two ways we can calculate the effect of article type: (1) a within-subjects comparison using reviewers' responses to both articles or (2) a between-subjects comparison using reviewers' responses to the randomly assigned first article. Between-subjects effects have less power than within-subjects effects, but we preregistered both within- and between-subjects analysis to address two concerns. First, a priori we were concerned that the length of our survey may lead to high drop-out after the first article, potentially hampering the within-subjects model. In reality, $99.43 \%$ of the reviewers provided data for both articles. Second, even if we did not have high attrition, a between-subjects comparison minimizes reviewers' awareness of the treatment. After the first article they have only experienced one condition. For these reasons, we estimated both a within-subjects and between-subjects effect of article type by running two different models for each survey question. Crossed effects 
Once reviewers were placed into a RR condition using keywords, they were then randomly assigned to matching condition (which of two comparison articles, same-author or same-journal) and a counterbalance condition (which of two orders to review the articles, RR 1st or $2 \mathrm{nd}$ ). This means that each article pair is crossed with matching and counterbalanced conditions. This allows us to estimate variation across matching and counterbalance conditions. Model 1: Within-subjects Model

In this model we estimated the overall within-subject effect of article type on each of our survey items. In our original preregistration (https://osf.io/a6xfj), we stated that we would include fixed effects for article type, matching scheme, counterbalance condition, the four interaction terms between these three predictors, and the discipline of each article pair. We also stated that we would include random effects for subject to account for the within-subjects design, an intercept for article pair, a random slope for article type by article pairs, and that our outcome measure would be a difference score. This model is impossible to estimate (as the difference score removes article type as a possible effect to estimate), and we realized this mistake when analyzing the first outcome. At that point, we updated the preregistration (https://osf.io/wjxsg) based on a comparison of fit between two different potential specifications of the within-subjects model, and the model that fit better was then used for all other outcomes (which had not been looked at when the preregistration was amended). This new model included a difference score outcome, fixed effects for article discipline, matching scheme, counterbalance condition, and the interaction between matching and counterbalance, and a random intercept for article pairs. The intercept of this model estimates the article type effect. See Supplementary Table 7 for full model specification.

Model 2: Between-subjects Model 
In this model we estimated the overall between-subject effect of article type on each of our survey items, using only survey responses to the first article rated by each reviewer. We modeled each survey response item as a function of the discipline, article type, matching scheme, and interaction of article type and matching scheme. These four terms plus the intercept resulted in a total of five fixed effect terms in the model. We included a random intercept for the article pair and a random slope of the article type by article pair. See Supplementary Table 8 for full model specification.

\section{Bayesian Estimation Methods}

We use Bayesian methods to estimate the mixed effects models. We assigned uninformative priors to all fixed effect parameters so that the results depend only on the data, similar to frequentist inference methods. One analyst (C.K.S.) ran the models in STAN (using the brms R package) using Hamiltonian Monte Carlo methods with four chains. ${ }^{39}$ A second analyst (K.M.E.) independently reproduced the results of Figure 1 in OpenBUGS and the reproduced results are identical to the ones reported in the paper.

We set weakly informative priors for both the between-subject and the within-subject model. We set priors of $\mathrm{N}(0,2)$ for the coefficients because the response scale is a 9-point scale ranging from -4 to 4 for fixed effects. Setting the standard deviation of the prior to 2 is conservative in that it places much of the likelihood around smaller changes in rating across conditions. And, we used half- $\mathrm{N}(0,2.5)$ priors for the priors of the hyperparameters to achieve partial pooling. We also performed sensitivity analyses to assess the extent to which our priors are affecting our estimates and found that the priors we use have no relevance to the results. For example, in the reproduction, we reparameterized the standard deviation parameters using the exponential function, and then assigned flat $\mathrm{N}(0,1000)$ priors for all parameters and found identical results. 
We used a number of different procedures to assess model fit and to check for convergence. To assess convergence we visually inspected the trace plots and Rhat values. Following evidence of model convergence, we assessed the fit of the models to the data through posterior predictive distributions. We created posterior predictive distributions for the global (overall average) article type parameter, its standard deviation, and the maximum and minimum values for article pairs. We compared these simulated values to the observed values in our dataset to determine if the model is accurately capturing different important facets of our data. For all models, we report the widely applicable information criterion (WAIC) (or leave-one-out (LOO) if it is suggested in place of WAIC by the Bayesian estimation program), parameter estimates, and $95 \%$ credible intervals.

\section{Preregistration Availability}

The preregistered design and analysis plan is available on OSF: https://osf.io/a6xfj and

\section{https://osf.io/wjxsg}

\section{Data Availability}

All data files are available on OSF: https://osf.io/aj4zr/

\section{Code Availability}

All files and scripts are available on OSF: $\underline{\text { https://osf.io/aj4zr/ }}$

\section{References}

1. Chambers, C. What's next for Registered Reports? Nature 573, 187-189 (2019).

2. Chambers, C. The registered reports revolution Lessons in cultural reform. Significance 16, 23-27 (2019).

3. Nosek, B. A. \& Lakens, D. Registered Reports: A Method to Increase the Credibility of Published Results. Soc. Psychol. 45, 137-141 (2014).

4. Nosek, B. A., Spies, J. R. \& Motyl, M. Scientific Utopia: II. Restructuring Incentives and 
Practices to Promote Truth Over Publishability. Perspect. Psychol. Sci. 7, 615-631 (2012).

5. Smith, R. Peer review: a flawed process at the heart of science and journals. J. R. Soc.

Med. 99, 178-182 (2006).

6. Fanelli, D. Negative results are disappearing from most disciplines and countries.

Scientometrics 90, 891-904 (2012).

7. Fanelli, D. "Positive" results increase down the hierarchy of the sciences. PloS One 5, e10068 (2010).

8. Franco, A., Malhotra, N. \& Simonovits, G. Publication bias in the social sciences: Unlocking the file drawer. Science 345, 1502-1505 (2014).

9. Dickersin, K. The existence of publication bias and risk factors for its occurrence. Jama 263, 1385-1389 (1990).

10. Mahoney, M. J. Publication prejudices: An experimental study of confirmatory bias in the peer review system. Cogn. Ther. Res. 1, 161-175 (1977).

11. Greenwald, A. G. Consequences of prejudice against the null hypothesis. Psychol. Bull. 82, 1-20 (1975).

12. Sterling, T. D. Publication Decisions and their Possible Effects on Inferences Drawn from Tests of Significance-or Vice Versa. J. Am. Stat. Assoc. 54, 30-34 (1959).

13. Makel, M. C., Plucker, J. A. \& Hegarty, B. Replications in Psychology Research: How Often Do They Really Occur? Perspect. Psychol. Sci. J. Assoc. Psychol. Sci. 7, 537-542 (2012).

14. Schmidt, S. Shall we Really do it Again? The Powerful Concept of Replication is Neglected in the Social Sciences. Rev. Gen. Psychol. 13, 90-100 (2009).

15. Makel, M. C. \& Plucker, J. A. Facts Are More Important Than Novelty. Educ. Res. (2014) doi:10/gcmc76.

16. Schimmack, U. The ironic effect of significant results on the credibility of multiple-study 
articles. Psychol. Methods 17, 551-566 (2012).

17. Giner-Sorolla, R. Science or Art? How Aesthetic Standards Grease the Way Through the Publication Bottleneck but Undermine Science. Perspect. Psychol. Sci. 7, 562-571 (2012).

18. Begley, C. G. \& Ellis, L. M. Raise standards for preclinical cancer research. Nature 483, $531-533$ (2012).

19. Prinz, F., Schlange, T. \& Asadullah, K. Believe it or not: how much can we rely on published data on potential drug targets? Nat. Rev. Drug Discov. 10, 712-712 (2011).

20. Camerer, C. F. et al. Evaluating replicability of laboratory experiments in economics. Science 351, 1433-1436 (2016).

21. Camerer, C. F. et al. Evaluating the replicability of social science experiments in Nature and Science between 2010 and 2015. Nat. Hum. Behav. 2, 637-644 (2018).

22. Klein, R. A. et al. Many Labs 2: Investigating Variation in Replicability Across Samples and Settings. Adv. Methods Pract. Psychol. Sci. 1, 443-490 (2018).

23. Klein, R. A. et al. Investigating Variation in Replicability: A “Many Labs" Replication Project. Soc. Psychol. 45, 142-152 (2014).

24. Open Science Collaboration. Estimating the reproducibility of psychological science. Science 349, aac4716-aac4716 (2015).

25. Ebersole, C. R. et al. Many Labs 3: Evaluating participant pool quality across the academic semester via replication. J. Exp. Soc. Psychol. 67, 68-82 (2016).

26. Allen, C. \& Mehler, D. M. A. Open science challenges, benefits and tips in early career and beyond. PLOS Biol. 17, e3000246 (2019).

27. Scheel, A. M., Schijen, M. \& Lakens, D. An excess of positive results: Comparing the standard Psychology literature with Registered Reports. https://osf.io/p6e9c (2020) doi:10.31234/osf.io/p6e9c. 
28. Hummer, L. T., Singleton Thorn, F., Nosek, B. A. \& Errington, T. M. Evaluating Registered Reports: A Naturalistic Comparative Study of Article Impact. https://osf.io/5y8w7 (2017) doi:10.31219/osf.io/5y8w7.

29. Cropley, A. Research as Artisanship versus Research as Generation of Novelty: The March to Nowhere. Creat. Res. J. (2018).

30. Baumeister, R. F. Charting the future of social psychology on stormy seas: Winners, losers, and recommendations. J. Exp. Soc. Psychol. 66, 153-158 (2016).

31. Nosek, B. A. \& Errington, T. M. The best time to argue about what a replication means? Before you do it. Nature 583, 518-520 (2020).

32. Gelman, A., Hill, J. \& Yajima, M. Why We (Usually) Don't Have to Worry About Multiple Comparisons. J. Res. Educ. Eff. 5, 189-211 (2012).

33. Epskamp, S. \& Nuijten, M. B. statcheck: Extract statistics from articles and recompute $p$ values. $R$ package version 1.3.1. (2018).

34. Hardwicke, T. E. \& loannidis, J. P. A. Mapping the universe of registered reports. Nat. Hum. Behav. (2018) doi:10/gf9dbb.

35. Chambers, C. D. \& Mellor, D. T. Protocol transparency is vital for registered reports. Nat. Hum. Behav. 2, 791-792 (2018).

36. John, L. K., Loewenstein, G. \& Prelec, D. Measuring the Prevalence of Questionable Research Practices With Incentives for Truth Telling. Psychol. Sci. 23, 524-532 (2012).

37. Simmons, J. P., Nelson, L. D. \& Simonsohn, U. False-Positive Psychology: Undisclosed Flexibility in Data Collection and Analysis Allows Presenting Anything as Significant. Psychol. Sci. 22, 1359-1366 (2011).

38. Nuijten, M. B., van Assen, M. A. L. M., Hartgerink, C. H. J., Epskamp, S. \& Wicherts, J. M. The Validity of the Tool "statcheck" in Discovering Statistical Reporting Inconsistencies. 
https://osf.io/tcxaj (2017) doi:10.31234/osf.io/tcxaj.

39. Stan Development Team. Stan Modeling Language Users Guide and Reference Manual.

(2020).

\section{Acknowledgements}

We thank Lilian Hummer for help with study planning, Alex Denis and Zach Loomas for help preparing survey materials, Brooke Bouza and Nick Buttrick for help with implementing the survey in Qualtrics, and Aurélien Allard for help coding the articles. This research was funded by grants from Arnold Ventures and James S. McDonnell Foundation (grant \# 220020498) and supported by the National Science Foundation Graduate Research Fellowship Program (grant \# 1247392 awarded to S.R.S). The funders had no role in study design, analysis, decision to publish, or preparation of the manuscript.

\section{Authors' Contributions}

Conceptualization:

Survey: C.K.S., T.M.E., B.A.N.

Article coding: S.R.S., J.G.B., S.V.

Data curation:

Survey: C.K.S.

Article coding: S.R.S., J.G.B.

Formal analysis:

Survey: C.K.S., K.M.E.

Article coding: S.V., S.R.S., J.G.B.

Investigation:

Survey: C.K.S., T.M.E.

Article coding: S.R.S., J.G.B., S.V.

Methodology:

Survey: C.K.S., T.M.E., K.M.E., B.A.N.

Article coding: S.R.S., J.G.B., S.V.

Software:

Article coding: S.R.S., J.G.B.

Visualization:

Survey: C.K.S.

Article coding: S.R.S., J.G.B.

Validation:

Survey: K.M.E.

Article coding: S.V.

Project administration: T.M.E.

Resources: T.M.E., F.S.T.

Supervision: T.M.E., B.A.N.

Funding acquisition: T.M.E., B.A.N.

Writing - original draft: C.K.S., T.M.E., K.M.E., B.A.N.

Writing - review \& editing: C.K.S., T.M.E., S.R.S., J.G.B., F.S.T., S.V., K.M.E., B.A.N. 


\section{Competing Interests}

T.M.E., C.K.S., and B.A.N. are employees of the nonprofit Center for Open Science (COS) that has a mission to increase openness, integrity, and reproducibility of research. COS offers support to journals, editors, and researchers in adopting and conducting Registered Reports. 
Figure 1. Posterior probability distributions for parameter estimates from within-subjects analysis using partial-pooling across the 19 outcomes comparing the difference of Registered Reports (RRs) and comparison articles. Black horizontal lines indicate 80\% (thick) and 95\% (thin) credible intervals and dots show the mean of the posteriors. Positive values indicate a performance advantage for RRs, negative values indicate a performance advantage for comparison articles. Outcomes are sorted from strongest to weakest performance advantage for RRs in the three evaluation groups: Before knowing study outcomes, after knowing study outcomes, and after finishing the paper.

\section{Evaluation before knowing study outcomes}

Methods rigor

Quality of methods

Amount will learn

Quality of question

Question \& methods aligned

Important research

Creativity of methods

Novelty of question

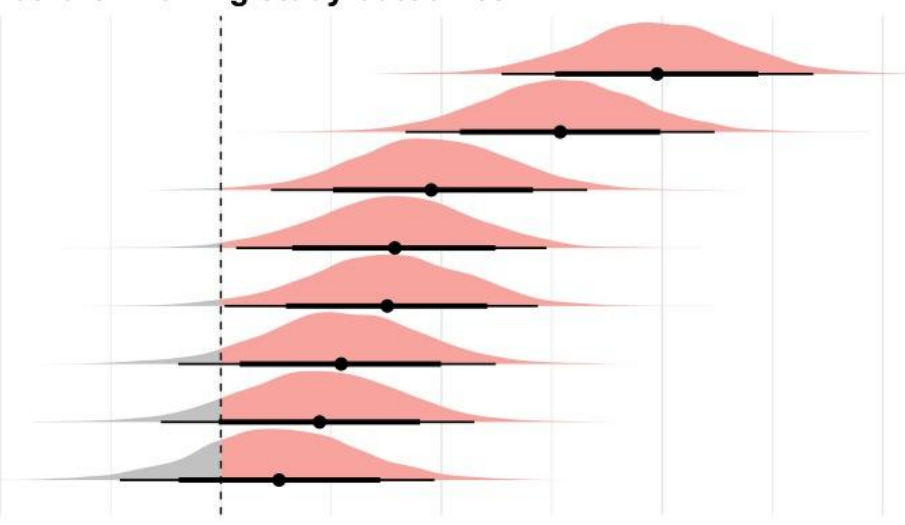

Evaluation after knowing study outcomes

Analysis rigor

Conclusions justified

Quality of results

Qualtiy of discussion

Amount learned

Innovative results

Important findings

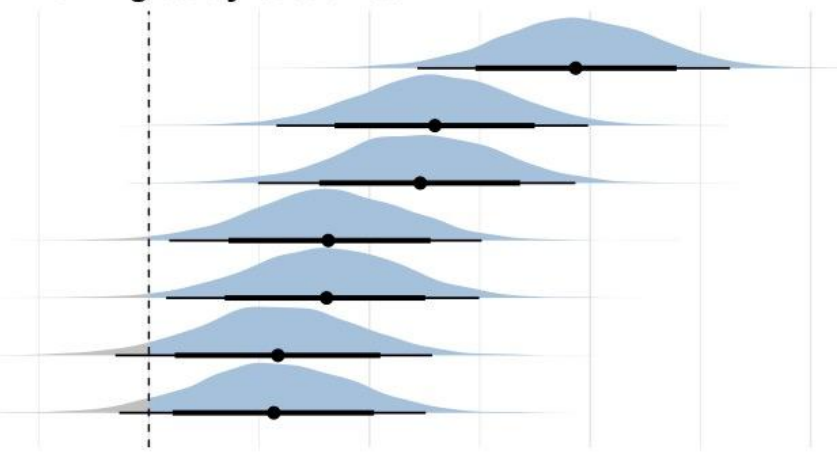

Evaluation after finishing the paper

Overall quality of paper

Important discoveries

Abstract \& findings aligned

Inspire new research

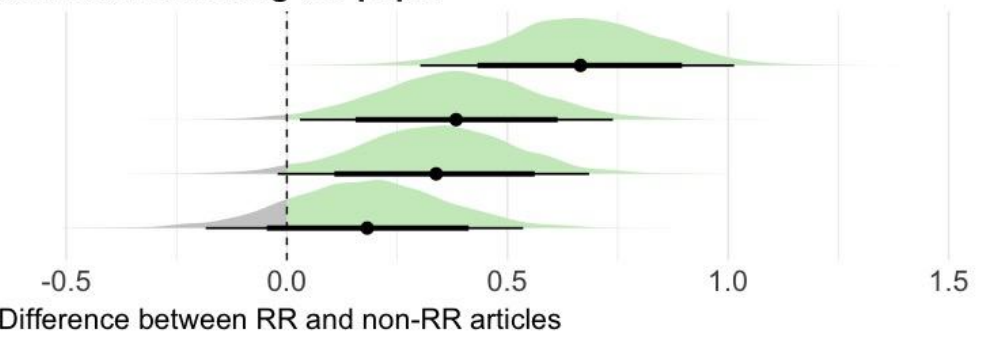


Table 1. Descriptive statistics of the last study of Registered Reports and comparison articles separated by author-based and journal-based

\begin{tabular}{lccc}
\hline & $\begin{array}{c}\text { Registered } \\
\text { Report }\end{array}$ & $\begin{array}{c}\text { Author-based } \\
\text { comparison }\end{array}$ & $\begin{array}{c}\text { Journal-based } \\
\text { comparison }\end{array}$ \\
\hline$N$ & 29 & 28 & 29 \\
\hline Statcheck results (last study) & & & $12(41 \%)$ \\
\hline $\begin{array}{l}\text { Detectable statistical tests } \\
\text { Errors }\end{array}$ & $12(41 \%)$ & $10(36 \%)$ & 0 \\
$M d$ & 0 & 0 & $0.58(1.00)$ \\
$M(S D)$ & $0.67(1.07)$ & $0.50(0.71)$ & 4 \\
Paper with $>0$ errors & 4 & 4 & 0 \\
Statistical significance decision errors & & & $0.17(0.58)$ \\
$M d$ & 0 & 0 & 1 \\
$M(S D)$ & $0.08(0.29)$ & $0.10(0.32)$ & \\
Papers with $>0$ decision errors & 1 & 1 & $2(7 \%)$ \\
\hline Open practices (last study) & & & $0(14 \%)$ \\
\hline Open materials & $17(59 \%)$ & $3(11 \%)$ & $(0 \%)$ \\
Open data & $25(86 \%)$ & $5(18 \%)$ & \\
Preregistration & $17(59 \%)$ & $2(7 \%)$ &
\end{tabular}

\section{Last study characteristics}

Sample size

$\begin{array}{lccc}M & 358.4 & 310.3 & 1150.0 \\ S D & 427.4 & 639.9 & 5119.0 \\ M d & 220 & 118 & 78 \\ \text { Sample justification (yes / no) } & 26 / 3 & 8 / 20 & 5 / 24 \\ \text { Difficult to reach population (yes / no) } & 1 / 28 & 6 / 22 & 6 / 23 \\ \text { Online data collection } & 13(45 \%) & 5(18 \%) & 4(14 \%) \\ \text { Experimental / non-experimental } & 24 / 5 & 18 / 10 & 22 / 7\end{array}$

Notes: Statcheck "papers with detectable statistical tests" includes papers for which statcheck detected at least one statistical result, namely correlations and $t, F, X 2, Z$, and $Q$ tests, reported in APA style; "errors" indicates all detected statistical reporting errors; "decision errors" indicates results reported as statistically significant when recomputed results were not, or vice versa. In principle, all Registered Reports were preregistered with the journal, but papers were not counted if a preregistration could not be found in an independent registry. 
Extended Data Fig. 1. Plot of correlations between all difference score outcome variables.

Correlation matrix of the 19 outcome variables with larger darker blue circles indicating stronger positive correlations than smaller lighter blue circles.

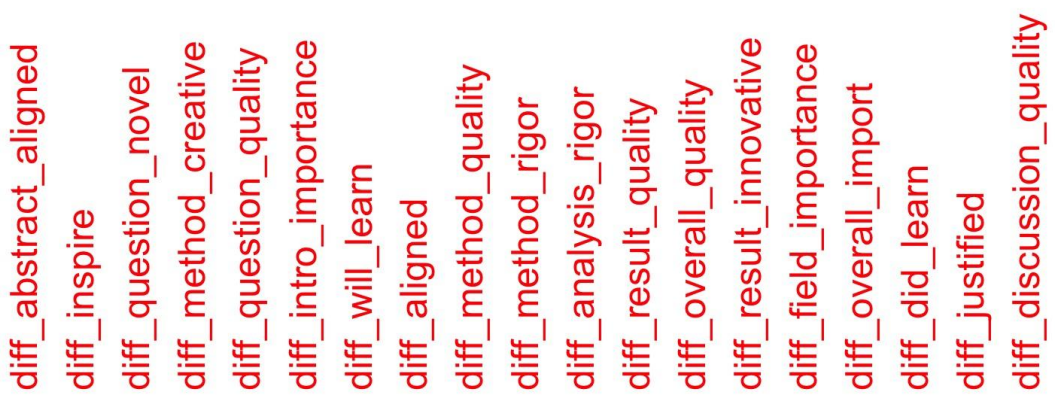

diff_abstract_aligned

diff_inspire

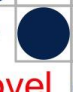
政

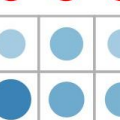

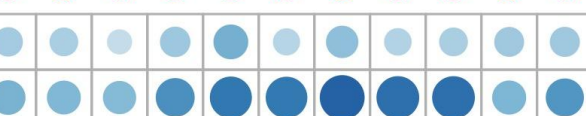
diff_question_novel diff_method_creative

diff_question_quality diff_intro_importance diff_will_learn
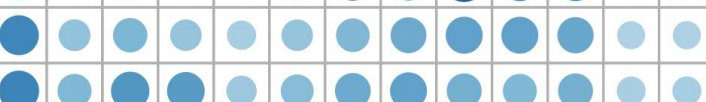
diff_aligned

diff_method_quality diff_method_rigor diff_analysis_rigor

diff_result_quality diff_overall_quality diff_result_innovative diff_field_importance diff_overall_import

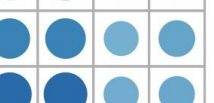

diff_did_learn diff_justified

diff_discussion_quality 
Extended Data Fig. 2. Posterior probability distributions for parameter estimates for each DV and each level of Familiar comparing the difference of RRs and comparison articles.

Horizontal lines indicate $80 \%$ (thick) and 95\% (thin) credible intervals and dots show the mean of the posteriors. Positive values indicate a performance advantage for RRs, negative values indicate a performance advantage for comparison articles.

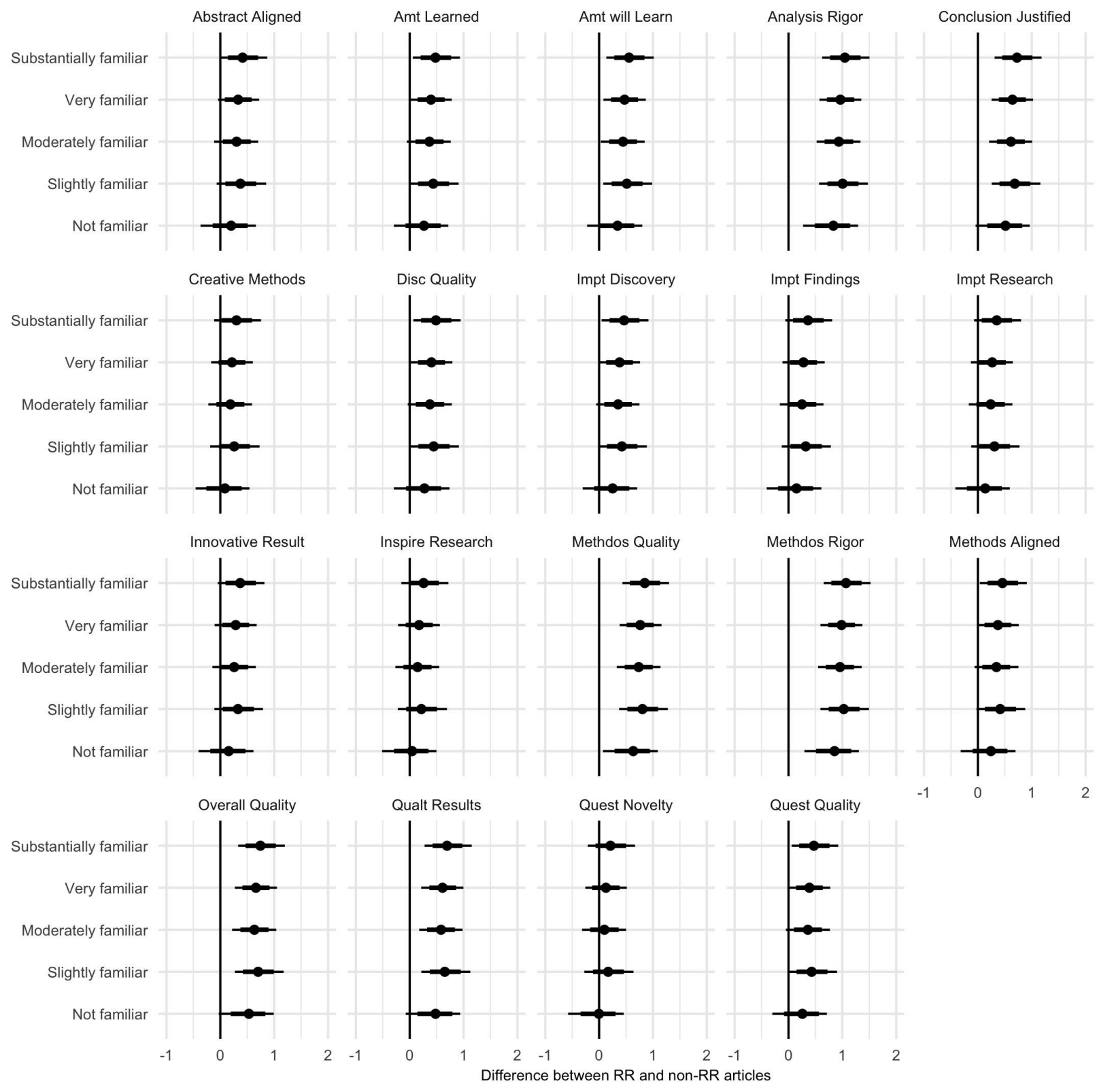


Extended Data Fig. 3. Posterior probability distributions for parameter estimates for each DV and each level of Improve comparing the difference of RRs and comparison articles.

Horizontal lines indicate $80 \%$ (thick) and 95\% (thin) credible intervals and dots show the mean of the posteriors. Positive values indicate a performance advantage for RRs, negative values indicate a performance advantage for comparison articles.
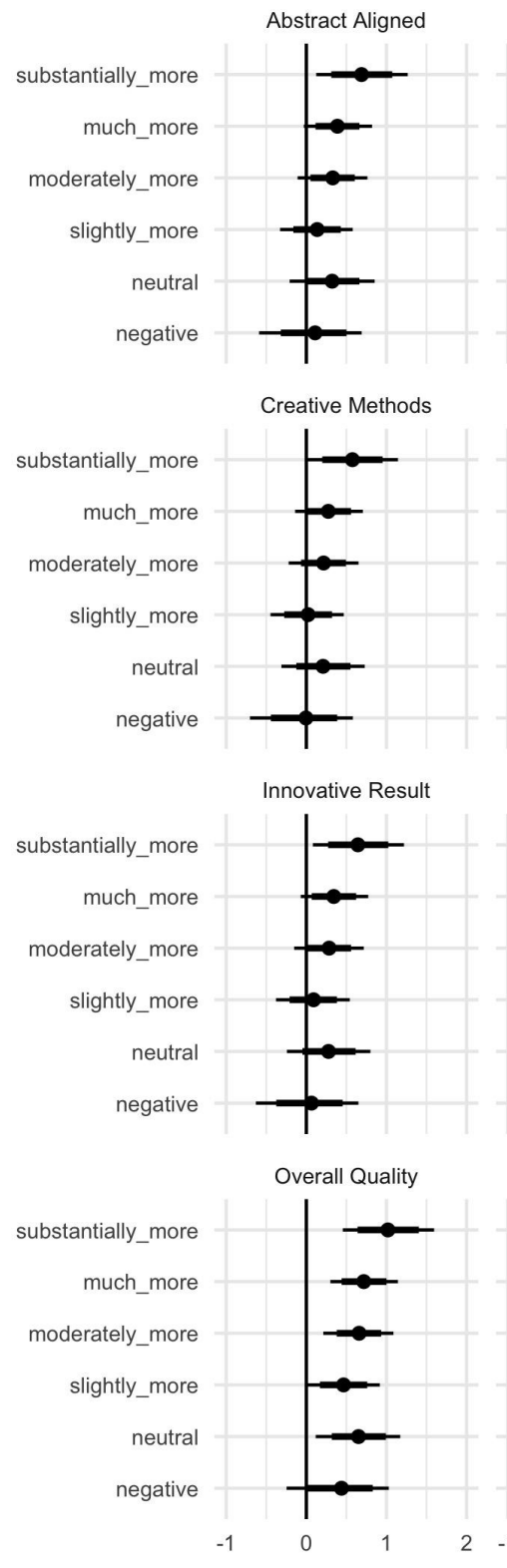

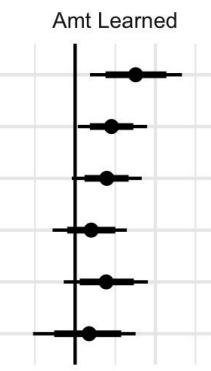

Disc Quality
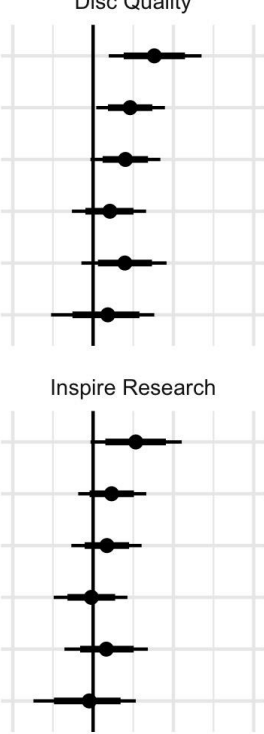

Qualt Results

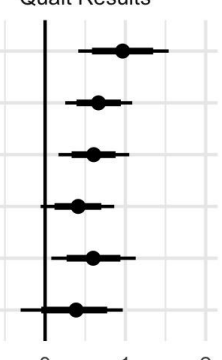

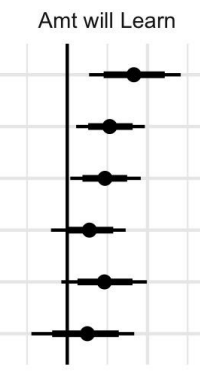

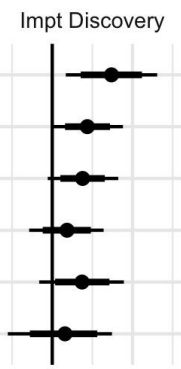

Methdos Quality

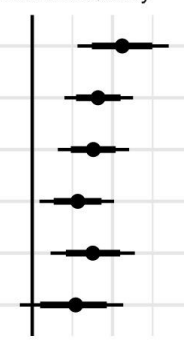

Quest Novelty

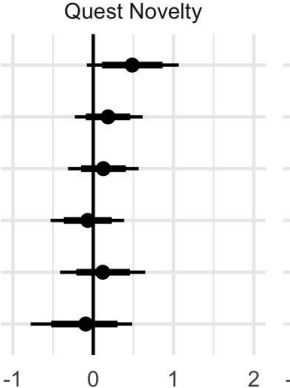

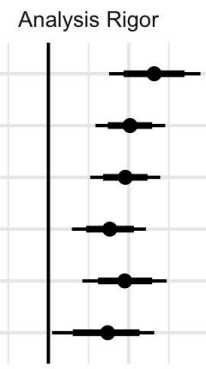

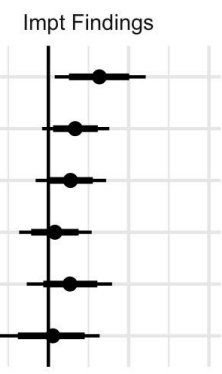

Methdos Rigor

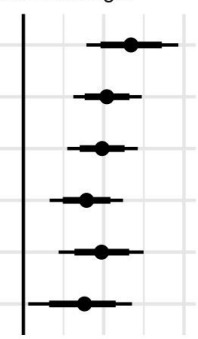

Quest Quality
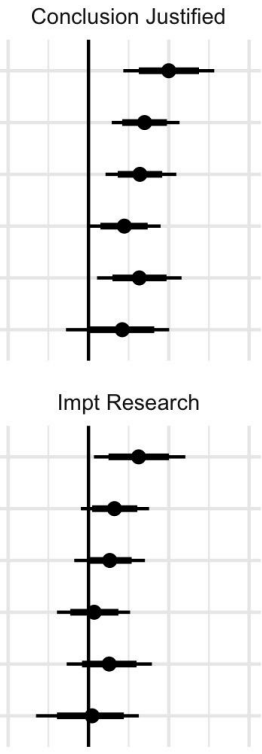

Methods Aligned

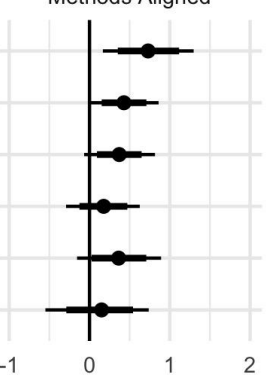

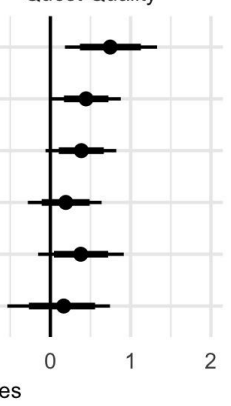


Extended Data Fig. 4. Posterior probability distributions for parameter estimates for each DV and each level of 'Guessed Right' comparing the difference of RRs and comparison articles.

Horizontal lines indicate $80 \%$ (thick) and $95 \%$ (thin) credible intervals and dots show the mean of the posteriors. Positive values indicate a performance advantage for RRs, negative values indicate a performance advantage for comparison articles.
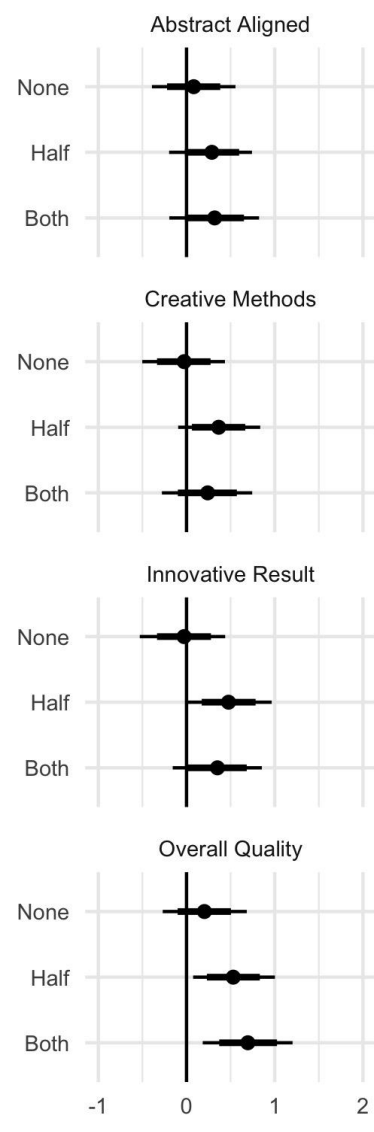

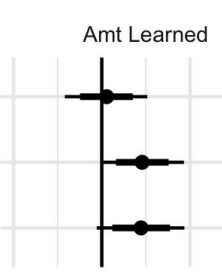

Disc Quality
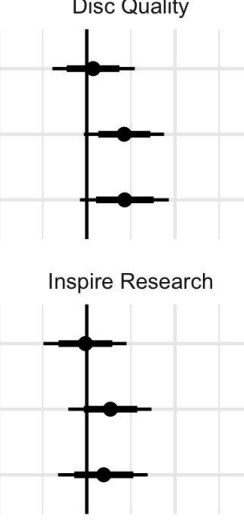

Qualt Results

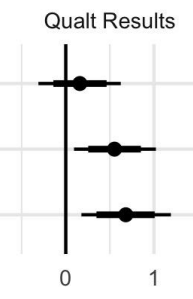

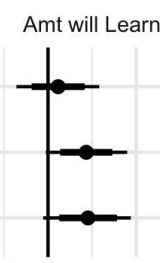

Impt Discovery

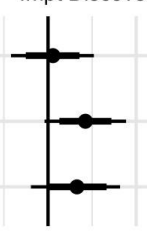

Methdos Quality

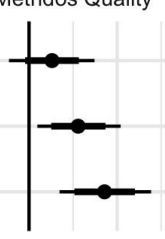

Quest Novelty

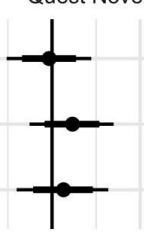

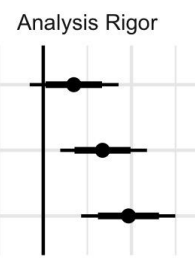
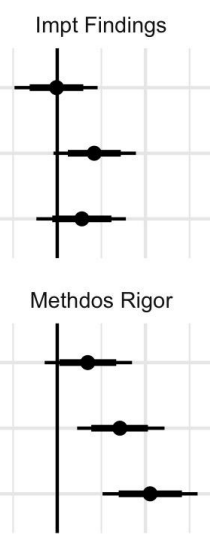

Quest Quality

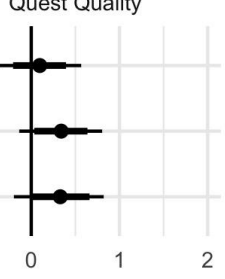

Conclusion Justified
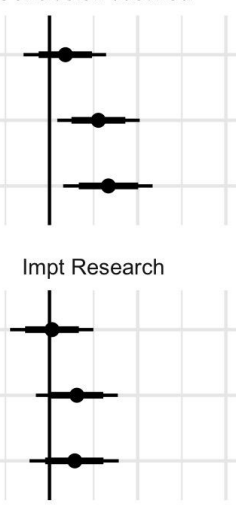

Methods Aligned

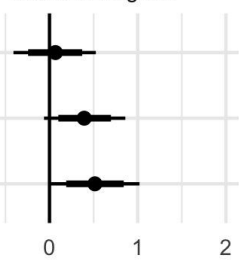




\section{Supplementary Information}

Initial Evidence of Research Quality of Registered Reports Compared to the Standard

Publishing Model

Soderberg, Errington, et al., 2020

\section{Table of Contents}

\section{Supplementary Methods}

Registered Report Article Selection

Comparison Article Selection

Article Coding

Reviewer Recruitment

Assigning reviewers to RR-comparison articles

Survey procedure

Survey Design 


\section{Supplementary Methods}

\section{Registered Report Article Selection}

Published RRs can be separated into two groups -- replications and novel research. RRs are perceived as particularly useful for replication research because the first stage peer review ensures expert feedback for effective administration of the study. Also, precommitment to the appropriateness of a replication design may reduce motivated reasoning to accept or reject replications based on the findings ${ }^{31}$. Thus, RRs provide a mechanism for publishing replication research, which are rarely published in the standard model ${ }^{13,14}$. However, the purpose of this study is to examine the relative benefits and costs of RRs. Because replications are rare in the standard model, conflating RRs with replications would interfere with the project aims. As such, we excluded replication RRs from this study and only examined RRs that investigated novel research questions. Supplementary Figure 1 shows the effect of our inclusion and exclusion criteria on our article sample of 29 RRs reporting novel research from psychology and neuroscience (Supplementary Table 5).

\section{Comparison Article Selection}

Journals and articles are not randomly assigned to be RRs. As a consequence, the value of this study hinges on identifying sensible comparison articles matched to RRs. To make causal inferences from quasi-experimental studies, an assumption of selection on observables must be made. This means that it is assumed that by conditioning on pre-treatment -observable variables that affect treatment assignment, in this case whether the article is an RR or not -- the dependence between assignment and outcome can be removed. Matching procedures are one way to remove pre-treatment differences between treatment and control groups. However, the success of matching procedures hinge on the extent to which such differences are observable and we have knowledge of what observable variables influence assignment. It is not known what factors influence the likelihood that an article becomes an RR, however we made some educated guesses and can avoid making strong causal claims.

If the same comparison article was identified through both methods, we planned to use that article as the author-based comparison article and identify another journal-based comparison article. This occurred 0 times when we implemented the article selection plan. We used a combination of exact matching on a few key variables and close matching on other variables in order of descending importance.

RRs published in Comprehensive Results in Social Psychology (CRSP) introduced a complication for our comparative investigation. CRSP published only RRs meaning that there are no articles published in standard format in the same journal for comparison. The benefit for including CRSP articles is maximizing the sample size of RRs for the investigation, the risk is losing some consistency of comparative study. We decided to include CRSP articles and identified comparison articles from another source--Social Psychology--a journal that publishes similar content as CRSP and published the first RRs in a special issue of replications ${ }^{3}$.

Each of the $29 \mathrm{RRs}$, with one exception, had matched articles for both selection criteria resulting in 57 comparison articles, for a total of 86 articles (Supplementary Table 5). 


\section{i. Journal-based comparison article matching procedure}

For the first comparison article for each RR, we selected an exact match on publishing journal as the primary criterion. We identified all empirical research articles, excluding other RRs, published in the same journal and same journal research section, if applicable (e.g., Frontiers in Psychology|Cognition), within one year before and after the RR first appeared online (i.e., ePublication date (ePub)). If multiple articles were identified we performed a keyword match (section a. Keyword match). If multiple articles were identified from the keyword match we performed a study number match (section $b$. Study number match). If multiple articles were identified from the study number match we performed a study design match (section $c$. Study design match). If multiple articles were identified from the study design match, we selected the comparison article with the highest keyword match rating. If there were ties, we randomly selected one of the remaining articles using http://random.orgl. If a single comparison article was selected for matching with multiple RRs, we planned to randomly pair it with a single matching RR and repeat comparison article selection for the other RRs disregarding the already matched comparison article. This occurred 4 times.

\section{ii. Author-based comparison article matching procedure}

For the second comparison article for each RR we selected an exact match on publishing author as the primary matching criterion. We focused on the first and last authors of each RR. Use of the last author for the senior member of the lab group or team is pervasive in neuroscience and common but not pervasive in psychology. To address this variability without introducing subjectivity in selection, if the corresponding author was neither the first or last author, then instead of "last author" we used "corresponding author". As an initial matching rule we identified all empirical research articles published by the first author of each RR within one year before and after the ePub (online publication) date of the RR. Of these articles, excluding other RRs, we selected those that appeared in a journal that had a Journal Impact Factor (JIF) score within two of the journal that published the RR (section d. Journal impact factor match). We then identified articles from these journals in which the author was also listed as the first author. If there were no 'first-author to first-author' matches, we repeated this approach using the last author. That is, all empirical research articles by the last author using the same criteria (+/- 1 year from ePub of RR and in a journal with a JIF score within two of the RR) were identified, and only those in which the author was also listed as the last/corresponding author was used. If there were no 'last-author to last-author' matches, we repeated this with articles published by the first author of the RR (+/- 1 year from ePub of RR and in a journal with a JIF score within two of the RR), but relaxed the matching criteria to include all articles, regardless of author position. If there were no 'first-author to any-author' matches, we repeated this approach with the last author. That is, all eligible articles published by the last author of the RR regardless of author position were included. If there were no 'last-author to any-author' matches, then the JIF matching criterion was relaxed (see section $d$ for details) and the process described above was repeated from the beginning. If no comparison articles are identified after that, there would be no author-based matched article for that RR. This occurred 1 time. 
If multiple comparison articles were identified then we identified the single comparison article by first performing a study number match (section b. Study number match), then if needed a study design match (section c. Study design match), and then if needed breaking tied articles by simple randomization.

\section{a. Keyword match}

We used article keywords to identify a comparison article that was most topically associated with the RR article. One of the authors (F.S.T.) generated a list of the keywords for each eligible comparison article associated with a unique identifier to anonymize the authors and articles. Other authors (C.K.S., K.M.E., T.M.E., B.A.N.) each rated the similarity of articles keywords to the RR keywords on a scale of 1 (not at all similar) to 10 (substantially similar). The top 5 keyword matched articles were selected for further matching criteria. If there were ties among the ranked articles preventing exactly 5 articles from being selected a simple randomization was used to identify 5 articles. For example, if the 4th and 5 th ranked articles had the same average rating, a simple randomization was used to identify which of these articles will be selected for further matching criteria.

\section{b. Study number match}

Articles vary in the number of reported studies. This evaluation focuses on a single study for most variables and most RRs report a single critical experiment. The number of studies could influence the perceived quality and rigor of accumulated evidence. As such, if an RR had one study, comparison articles with one study was selected. If a RR has multiple studies, we selected comparison articles that reported within two studies of the number reported in the RR. If there were no eligible articles, the parameters for number of studies for a matched article was relaxed by incrementally increasing the number of studies by one, until one or more comparison articles was identified. For example, to find matched articles for a RR with five studies, all articles that contained three to seven studies were identified; however, if no comparison articles were identified, then this was relaxed to include two to eight studies, and so on. This criterion either identified one matched article, or multiple articles that were used for further matching criteria.

\section{c. Study design match}

The study design of the RR was categorized as either observational or experimental and comparison articles of the same type were eligible for selection. If there were no matches then this criterion was not used. This criterion either identified one matched article, or multiple articles that were used for further matching criteria.

\section{d. Journal impact factor match}

Despite well-known limitations of Journal Impact Factor (JIF), it is a simple heuristic for identifying journal prestige as a selection criterion. The strength of association between prestige and quality is contested, so we apply it self-consciously to match articles by the same authors on relative prestige of the publication. The Web of Science JIF of the RR was determined using the most recently available score. Comparison articles published in journals with JIF scores 
within two points greater or less than the JIF score of the RR were identified. If necessary, this criterion was relaxed to expand the eligible journals by incrementally increasing the upper bound JIF score by one. For example, to find comparison articles for an RR published in a journal with a JIF score of 4.279 , all articles published in journals with a JIF score between 2.279 and 6.279 were identified. If no comparison articles were identified, then this was relaxed to include articles published in journals with a JIF score between 1.279 and 7.279, and so on. RRs published in CRSP did not have an assigned JIF score because the first article was published in 2016. As such, we used a JIF score of 1.12 that was determined from Dimensions (Digital Science) citation and publication data (2016: 4 citations, 8 publications; 2017: 24 citations, 17 publications).

\section{Article Coding}

\section{i. Human coding procedure.}

Coders included a team of four psychologists (faculty, post-docs, and graduate students) with expertise in metascience and experience in social and personality research. Each article was randomly assigned and independently coded by two people. The variables coded are described below and additional details and instructions are available in the preregistration (osf.io/maisw).

- Sample size: This was defined as the number of participants (or individual observations) included in a study. As a rule of thumb, we used the number of participants initially in the study, even if participants were excluded for various reasons (e.g., malfunctioning equipment, failure to follow instructions). The exception to this was situations in which the initial sample is much larger than the final sample (e.g., at least 2-3 times the final number of participants), usually because the initial sample was a broadsweep (e.g., mass testing sessions in Introductory Psychology) to identify participants who met the study criteria.

- Difficult to reach populations: This was defined as "populations [that] may be hard to reach because of their physical or social locations (e.g., remote geographical location, social elites), but they may also be hard to reach because they are vulnerable (i.e., disenfranchised, subject to discrimination or stigma) . .." $)^{40}$. This was coded as yes or no.

- Sample justification: This was defined as any justification for the sample size-regardless of the quality of the justification. This was coded as yes or no.

- Study design: Coders indicated whether the design was experimental, non-experimental, or something else using the following operationalizations

- Experimental: studies where at least one variable was manipulated by the experimenter.

- Non-experimental: studies where no variables were manipulated by the experimenter.

- Something else: this was selected if neither experimental or non-experimental properly described the study.

- Primary result: This was defined as the statistical test result(s) for the primary outcome of the last study. 
We used the following criteria to determine whether the coders disagreed on a certain coded variable:

- For sample size, absolute differences greater than $10 \%$ of the larger value were reevaluated by a third coder; for absolute differences smaller than $10 \%$, the average between both coders' estimate was the final reported value.

- For all other variables (except Primary Result), which were categorical, coders agreed when the two answers matched exactly.

Coders also attempted to extract the primary result for the last study, but this process was difficult and the coders expressed low confidence in their results. Review by a third coder confirmed this perception: the extracted primary result was seldom similar between coders, making it impossible to use the data.

ii. Open practices and online data collection coding procedure.

To identify articles that reported open practices (i.e., preregistration, open materials, and open data) or that collected data online, we used a semi-automated procedure. Variables were operationalized using the following criteria:

- Preregistration: The authors claim to have preregistered some portion of a study and include a link to a preregistration.

- Open materials: The authors claim to have shared study materials and include a link to materials or claim that the study materials are included in the supplemental materials.

- Open data: The authors claim that the data are publicly available and include a link to the dataset.

- Online data collection: The authors state the study was conducted online or reference a commonly used online survey platform.

For each variable, we coded whether a) no studies met the criteria, b) all studies met the criteria, c) the last (or only) study met the criteria, or d) a study that was not the last study met the criteria. For the open practices (preregistration, open materials, and open data), we coded whether a link was included in the paper, not whether the link was currently active or actually contained the information so claimed.

We scraped and converted text from the pdf file of each article to workable text files in $\mathrm{R}^{41}$ using the trickypdf ${ }^{42}$ and $x \mathrm{xl} 2^{43}$ packages. This process is imperfect, yet provides fairly accurate plain-text versions of the articles. Some potential issues with this method include the inaccurate recognition of special characters, which was not expected to impact these activities, and reading words split between two lines being as unconnected strings, which could have had minor impact on these activities. Following text scraping, we analyzed the text files to identify search terms and patterns potentially indicating the presence of a variable of interest (see the preregistration for additional detail osf.io/nb4k5/). Articles flagged by this procedure were screened by a researcher to confirm whether the article met the criteria for any of the variables of interest. To do so, a Shiny app was used to extract and display all paragraphs containing relevant text strings for the researcher to review. If it was unclear from the extracted text whether the article met the criteria, the researcher examined the full-text article.

iii. statcheck errors.

We used the statcheck $R$ package ${ }^{33}$ to extract test-statistics and recompute $p$-values to identify misreported $p$-values for tests reported in APA-style. We analyzed both the full-text pdf files for each paper and pdf files that included only the Results/Discussion sections of the last 
study reported in each paper (and general discussion). Thus, we searched for errors in the entire paper and errors specifically in the last study. Results from the analysis of the Results/Discussion sections of the last study reported in each paper (and general discussion) are reported in the main text. Results from the analysis of the full-text are reported in the Supplementary Table 9 below.

\section{Reviewer Recruitment}

Our most substantial barrier to effective execution of this study was recruiting reviewers to invest in completing two article reviews. Nevertheless, we believed that the high investment in that sampling was important for maximizing the information value of the accumulated evidence. Each review involved reading the full article and providing quantitative and qualitative review of features of the article. Because of expected variability in subjective assessment, we planned to recruit a sample of at least 360 reviewers to each evaluate two of a total sample of 90 articles ( $1 / 3$ RRs, $2 / 3$ comparison articles) for a total of 720 article reviews. Below we provide details of the sample selection process and the survey and coding schemes.

This project design is multi-level -- the number of papers to review (RRs and paired comparisons) and the number of peer reviewers reviewing those articles. That is, reviewers are nested within articles. The total number of articles (29) is fixed as the existing population of RRs reporting novel research. As such, the maximum attainable power is heavily influenced on the effect size and the proportion of the random variation due to the between-article variance ${ }^{35}$. If there is high between-article variance, increasing the number of reviewers will not have a substantial impact on power.

\section{Assigning reviewers to RR-comparison articles}

We aimed to maximize the match between reviewer expertise and the subject of the articles that they review. Simultaneously, we aimed to minimize selection bias of authors selecting articles that they know or wish to read. To balance these interests, reviewers first identified the discipline that best matches their expertise -- cognitive psychology, neuroscience, or social-personality psychology. With social-personality psychology, which represented 18 of the 29 article pairs, we presented five sub-disciplines -- media/applied psychology, group processes and intergroup relations, positive psychology, personality psychology, or attitudes and social cognition. Then, we presented them with a table of keywords. Each row was filled with the keywords for each RR and two matched comparison articles in their discipline. Supplementary Table 9 shows the keywords groups. Keywords that directly indicated preregistration, Registered Reports, or other author identifying terms were removed. Potential reviewers selected which of the keyword lists matches most closely with their research expertise. If they ultimately consented to participate, reviewers were then assigned to that RR and a randomly selected one of the two comparison articles. The use of keywords balances the desire to match expertise with the desire to avoid undesirable selection biases.

It was not necessary for us to have perfectly balanced sample sizes across each RR article pair; however the more extreme the sample size imbalance the more the results may be driven by a few article pairs. Our preregistration specified that, based on the number of respondents, we could remove some keyword options, and thus RR and comparison articles, 
during the course of the study. We did this on two occasions, removing keyword options that had more than 12 responses at the time we checked. The first keyword removal happened based on data from 207 participants and removed keywords for 5 article options (RR11, RR19, RR35, RR37, RR40). The next keyword removal happened based on data from 337 participants and removed keywords for an additional 9 article options (RR01, RR05, RR07, RR09, RR10, RR20, RR36, RR39, RR41). The removal of keywords during the experiment was incorporated into our within and between subjects models by including a fixed effect for whether participants encountered the original set of keywords or a reduced set, as outlined in our amended preregistration (https://osf.io/wjxsg). Comparisons of model estimates for the three different potential keyword models can be found in the supplement for our within and between subjects models.

\section{Survey procedure}

Reviewers received a link to the study via email. Reviewers that were recruited by general outreach methods read a brief description of the study and, if they agreed to participate, first self-identified the discipline most closely matching their expertise. Then, they selected which of the keyword lists best matched their expertise (Supplementary Table 10). Reviewers were assigned to the RR-comparison articles combo that they selected and then randomly assigned to which comparison article they would review. Reviewers completed the survey measures for one article before being presented with the survey measures for the second article. Reviewers were randomly assigned to receive the $\mathrm{RR}$ or matched comparison article first. After completing the articles, reviewers completed a short survey of their demographic characteristics and knowledge and beliefs about RRs and publishing.

\section{Survey Design}

i. 'Introduction and Methods' measures

After review of an article's Introduction and Methods sections the following questions were presented for each study for each reviewer:

1. What is the overall quality of the last study's research question?

2. What is the overall quality of the last study's methodology?

3. How rigorous is the methodology of the last study?

4. Regardless of what results will be observed, what is the overall importance of the research described in the last study?

5. How novel is the research question of the last study?

6. How creative is the methodology of the last study?

7. How much will we learn from the last study?

8. How aligned is the research question and the methodology of the last study?

\section{ii. 'Results and Discussion' measures}

After review of an article's Results and Discussion sections the following questions were presented for each study for each reviewer:

1. How rigorous is the analysis strategy of the last study for addressing the article's research question(s)? 
2. How would you describe the overall importance of the last study's findings?

3. How innovative are the results described in the last study?

4. How much did we learn from the last study?

5. How justified are the conclusions based on the article's results?

6. What is the overall quality of the last study's results?

7. What is the overall quality of the last study's discussion?

iii. 'Abstract' measures

After review of an article's Abstract the following questions were presented for each study for each reviewer:

1. How aligned is the abstract with the article's findings?

2. To what extent did the research produce important discoveries?

3. How much will this research inspire new research?

4. What is the overall quality of this research? 


\section{Supplementary Discussion}

This observational study was designed to evaluate potential benefits and costs of RRs compared to the standard publishing model. We first generated hypotheses for how RRs and standard papers may differ from one another and then used these hypotheses to guide development of the systematic peer review survey described above.

RRs emerged as a solution to some of the perceived problems with the traditional peer review and publication model. Some benefits of RRs may not require empirical demonstration, but others are hypotheses and empirically testable. Even if some benefits of RRs are definitive when considered in isolation, it is possible that other costs outweigh those benefits when considering the overall effect on research efficiency. Moreover, there is a difference between theory and practice. RRs might definitively protect against publication bias in theory, but fail to do so in practice because of weaknesses in implementation.

Potential Benefit: Improved rigor of methodologies and importance of research questions. There are at least two ways that RRs could increase the rigor and importance of the research. First, reviewers will not be distracted by the findings at the cost of observing the strengths and weaknesses of the methodology. For example, if a positive effect is observed, reviewers might overlook the fact that the study had a very small sample size, whereas, without knowing the result, the small sample size could be seen as an important limitation. Second, in RRs, reviewers' expert feedback can improve the methodology. In the standard model, identification of methodological weaknesses comes too late -- the studies have been completed. With RRs, identification of methodological weaknesses can spur revision and improvement to maximize the importance and potential impact of the study. There is initial evidence that the ultimate acceptance rate for RRs is higher than traditional papers ${ }^{28}$, perhaps because of the opportunity for methodological improvements during review. This might improve research efficiency and reduce reviewer fatigue, but examining publication statistics are not part of the present investigation.

Potential Benefit: More transparent and comprehensively described methodology and analysis strategies. RRs may also be associated with more transparent methodology than the standard model. The first stage of review directly evaluates the quality of the methodology and analysis plan. This may motivate reviewers to demand, and authors to express, the methods as completely as possible. In the standard model, it may be easier to overlook weak specification of methods because of greater attention to the findings. More completely described methods could improve reproducibility by making it easier for researchers to conduct effective replications.

Potential Cost: Polluting the literature with poorly done, uninformative studies. Things go wrong in research. Researchers sometimes fail to properly execute the methodology rendering the result uninformative for testing the hypothesis or even providing any useful data. A risk of pre-commitment to publication is that researchers will become more lackadaisical in proper execution of the methods. Or, even when they are doing their best, errors might occur and get published anyway. RRs address this possibility by having a second stage of peer review to evaluate the quality of execution -- such as using indicators of competent execution 
independent of the key outcomes. But, the extent to which this is performed and effective in practice is unknown.

Potential Cost: Less risk taking and innovation. A potential advantage of the standard publishing model compared with RRs is that authors can conduct studies on any idea that they wish. Researchers can pursue high-risk/high-reward strategies that result in many failures, but a singular success could make that entire program of research worthwhile. These high-risk strategies may be less likely in RR publishing formats. When decisions are made up front about the value and potential impact of research, authors and reviewers may become more conservative about the questions that they test. For example, hypotheses judged to have very low odds at the outset may be rejected by reviewers at the first stage of review based on assessed implausibility. The appropriate level of risk tolerance for research efficiency is not easy to assess, but it is worth investigating how different publishing models impact risk tolerance for low odds hypotheses.

Potential Cost: Less novelty or creativity. Related to risk taking, authors and reviewers may be more comfortable proposing and accepting paradigmatic methodologies that have a strong preexisting basis of evidence rather than embracing novel or creative methodologies or research questions. RRs have a mechanism for promoting creativity and risk-taking by allowing preliminary data for first stage review to demonstrate proof of concept. However, it is not clear if this results in similar risk-taking and creativity between traditional and RR publishing formats.

Potential Cost: Less interesting papers. Another potential cost of RRs is less interesting papers compared with the standard model. A common belief is that scholarly articles are most useful if they express what was learned rather than how the researcher got there ${ }^{30}$. Given the omnipresence of positive results in the published literature, a just-world hypothesis is that showing lack of support for a hypothesis or negative results will be less interesting or impactful and so such papers are not written, submitted, or published. However, there is little systematic evidence identifying the interestingness of papers contingent on the observed results. Initial evidence against the just-world hypothesis is that an initial investigation of altmetric and citation impact suggests no difference or slightly better performance for RRs compared to similar articles published at the same time in the same journals ${ }^{28}$. Nevertheless, citations are an indirect indicator of "interestingness" at best.

There are other potential benefits and costs that could be associated with RRs compared to the standard publishing model. Some potentially important ones that we did not examine but are worth addressing in other research include:

Potential Benefit: Minimizing publication bias. Positive results are much more likely to be published than negative results ${ }^{5-7}$. The bias against negative results appears to be a consequence of both editors and reviewers finding them less compelling for publication and from authors themselves not trying to publish them ${ }^{8-12}$. RRs eliminate this bias by making an initial commitment to publish without knowing the results. Nevertheless, RRs may not entirely eliminate this bias if the second stage of review is influenced by the observed outcomes. In RRs, the second stage of review is supposed to focus on indicators of competent execution and appropriateness of interpretations and conclusions. It is possible that RRs receive greater 
scrutiny and ultimate rejection for negative than positive results. This hypothesis was supported in two prior investigations ${ }^{26,27}$.

Potential Benefit: Increase clarity of confirmatory versus exploratory research.

Mistaking exploratory research that generates hypotheses for confirmatory research that tests hypotheses can contribute to inflation of false positives and exaggerated effect sizes. RRs should clearly distinguish confirmatory tests and exploratory discoveries by having authors commit to their analysis plan at initial acceptance and report all prespecified analyses in the final report. Any analyses beyond the pre-specified plan can be clearly labeled as exploratory to promote caution in drawing conclusions with those findings by authors and readers. Realization of this benefit requires that the RR process and reporting adheres to the plan specified and accepted during the first stage of RR review.

Potential Benefit: More explicit recognition of limitations. RRs may also encourage more explicit discussion of the limitations of the findings compared with the standard model. This could occur for two reasons. First, the first stage review process may identify more of the strengths and limits of the methodology, and those limitations may be a more explicit part of the paper and its conclusions. Second, RRs may decrease pressure for clean narratives that underreport exceptions, qualifications, or ill-fitting evidence. The prespecified analysis plan may make authors more adherent to planned analyses and more open to considering and discussing qualifications to the conclusions.

Potential Cost: Less exploration of the observed data. A proposed benefit of RRs is making clear the distinction between confirmatory and exploratory research. A potential cost of that clarity is that authors may be more reluctant to conduct and report exploratory analyses. Indeed, in the traditional publishing model, there are incentives for having a strong a priori hypothesis-testing narrative relegating exploratory investigations to perceived lower status. Alternatively, making the distinction explicit could increase the perceived value of exploratory analysis and help authors embrace it as a valued part of the research process. 


\section{Supplementary Figures}

\section{Supplementary Figure 1}

Flow chart of Registered Report articles based on inclusion and exclusion criteria.

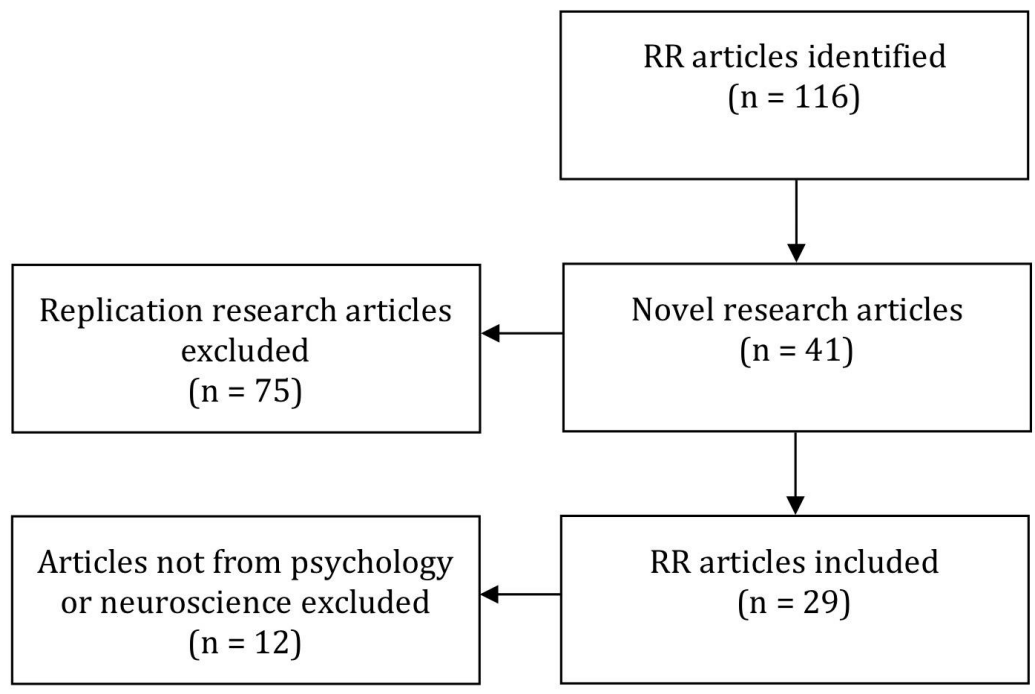




\section{Supplementary Figure 2}

Posterior probability distributions for parameter estimates from the ML within-subjects analyses comparing the difference of Registered Reports (RRs). For each outcome, the posteriors for 5 different sets of priors are shown. Horizontal lines indicate $80 \%$ (thick) and $95 \%$ (thin) credible intervals and dots show the mean of the posteriors. Positive values indicate a performance advantage for RRs, negative values indicate a performance advantage for comparison articles.

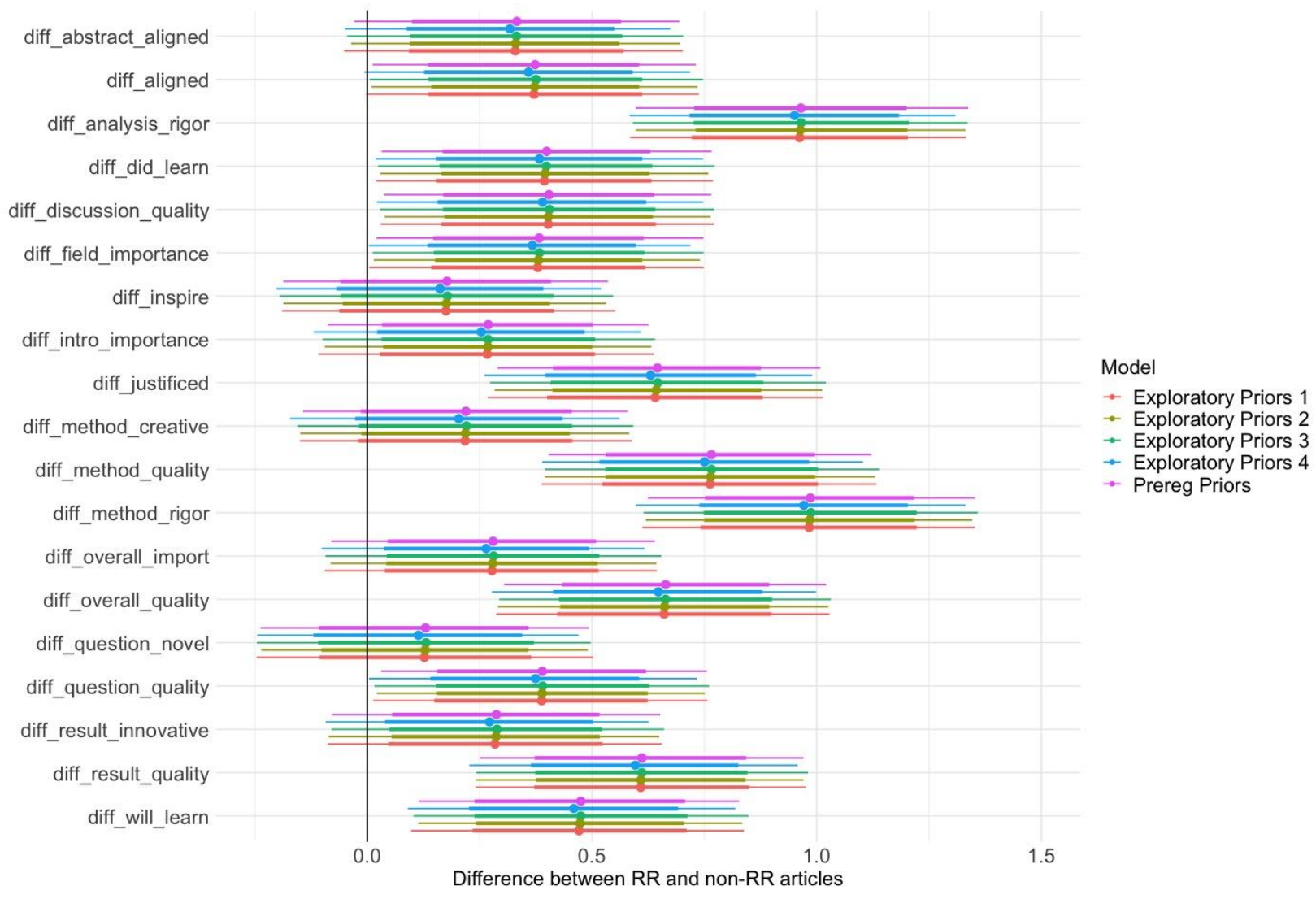




\section{Supplementary Figure 3}

Posterior probability distributions for parameter estimates from within-subjects analyses comparing the difference of Registered Reports (RRs) and comparison articles for a model that has no pooling across outcome and the multilevel model that includes partial pooling across outcomes. Horizontal lines indicate $80 \%$ (thick) and $95 \%$ (thin) credible intervals and dots show the mean of the posteriors. Positive values indicate a performance advantage for RRs, negative values indicate a performance advantage for comparison articles.

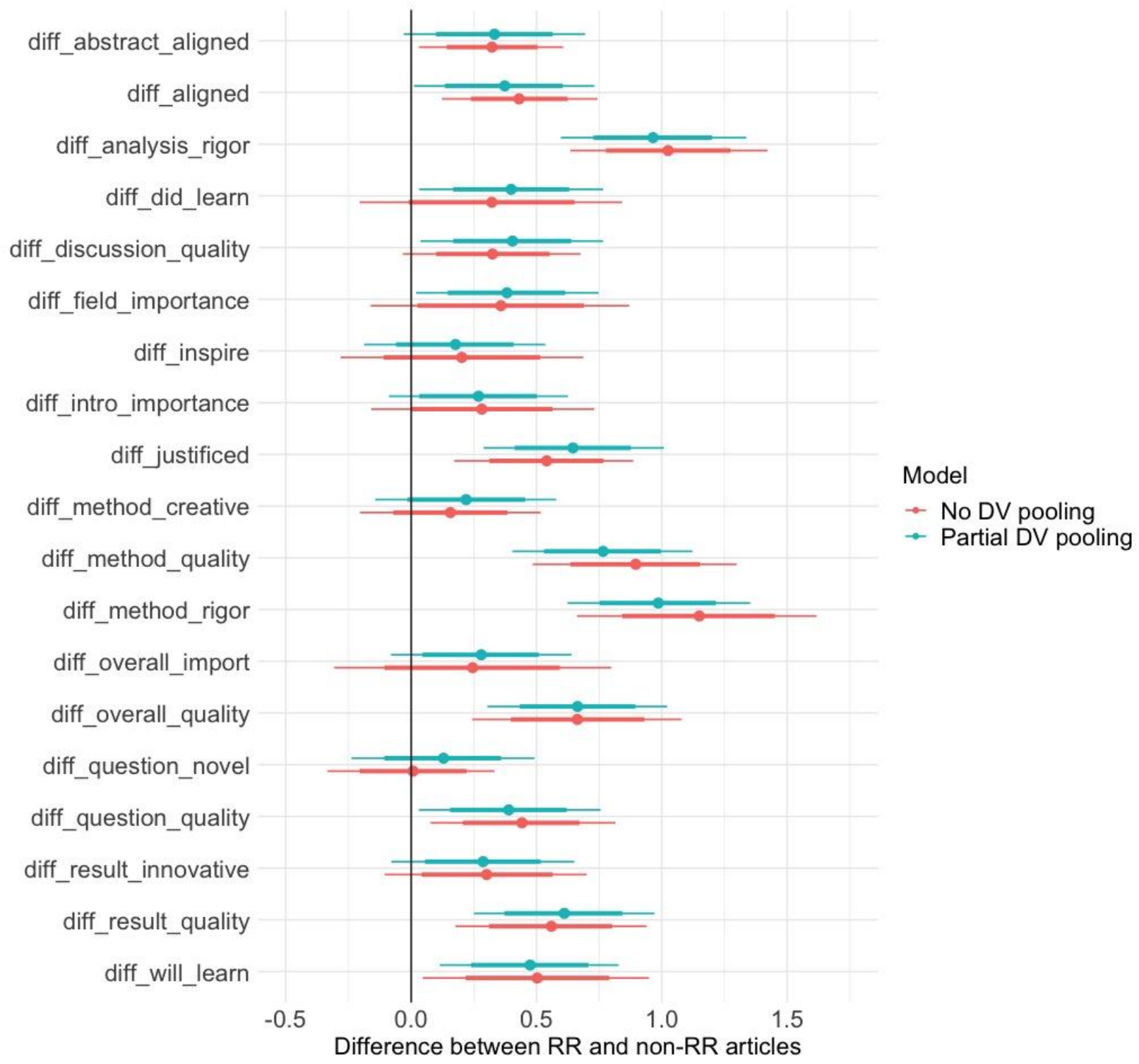




\section{Supplementary Figure 4}

Posterior probability distributions for parameter estimates from between-subjects analysis comparing the difference of Registered Reports (RRs) and comparison articles for each outcome variable separately. For each outcome, a separate estimate is plotted for a model that only uses subject who saw the initial, complete set of keywords (red lines), later subjects who saw a reduced set of keywords (blue lines), or a model that pooled all participants but includes a fixed effect for whether they saw the full set or reduced set of keywords (green line). Horizontal lines indicate $80 \%$ (thick) and $95 \%$ (thin) credible intervals and dots show the mean of the posteriors. Positive values indicate a performance advantage for RRs, negative values indicate a performance advantage for comparison articles.

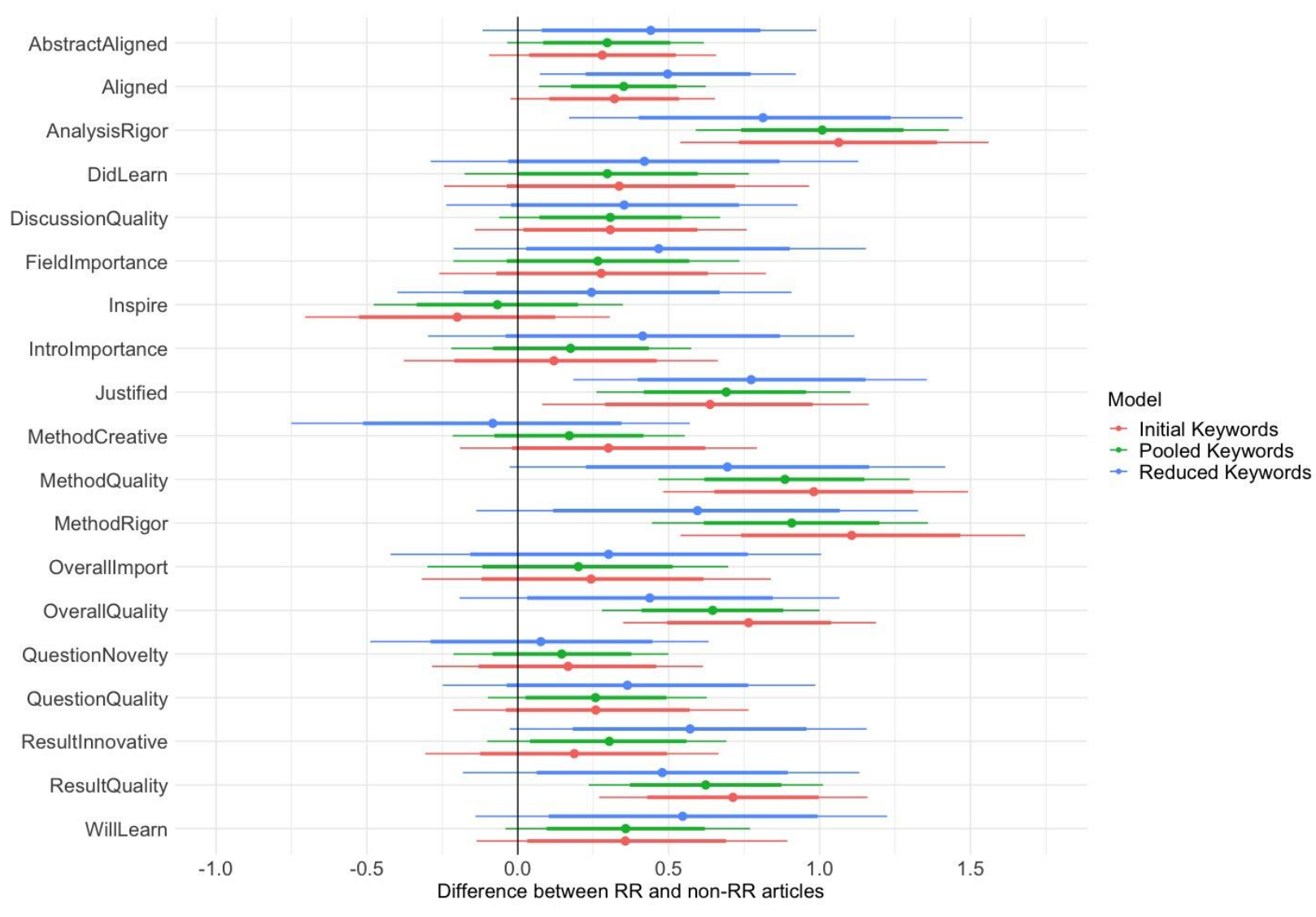




\section{Supplementary Figure 5}

Posterior probability distributions for parameter estimates from within-subjects analysis comparing the difference of Registered Reports (RRs) and comparison articles for each outcome variable separately. For each outcome, a separate estimate is plotted for a model that only uses subject who saw the initial, complete set of keywords (red lines), later subjects who saw a reduced set of keywords (blue lines), or a model that pooled all participants but includes a fixed effect for whether they saw the full set or reduced set of keywords (green line). Horizontal lines indicate $80 \%$ (thick) and 95\% (thin) credible intervals and dots show the mean of the posteriors. Positive values indicate a performance advantage for RRs, negative values indicate a performance advantage for comparison articles.

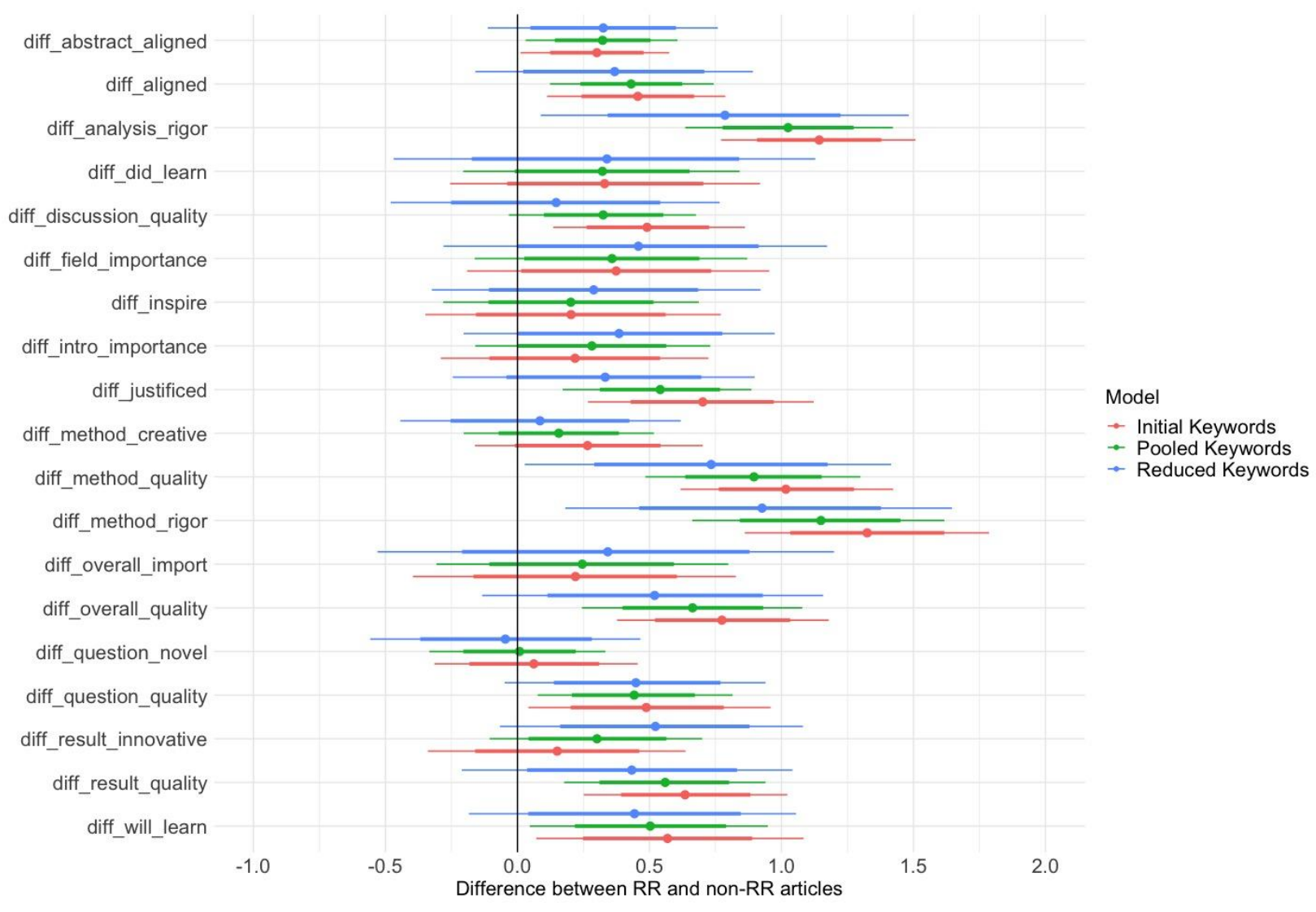




\section{Supplementary Figure 6}

Posterior probability distributions for parameter estimates from between-subjects analyses comparing the difference of Registered Reports (RRs) and comparison articles for a model that has no pooling across outcome and the multilevel model that includes partial pooling across outcomes. Horizontal lines indicate $80 \%$ (thick) and $95 \%$ (thin) credible intervals and dots show the mean of the posteriors. Positive values indicate a performance advantage for RRs, negative values indicate a performance advantage for comparison articles.

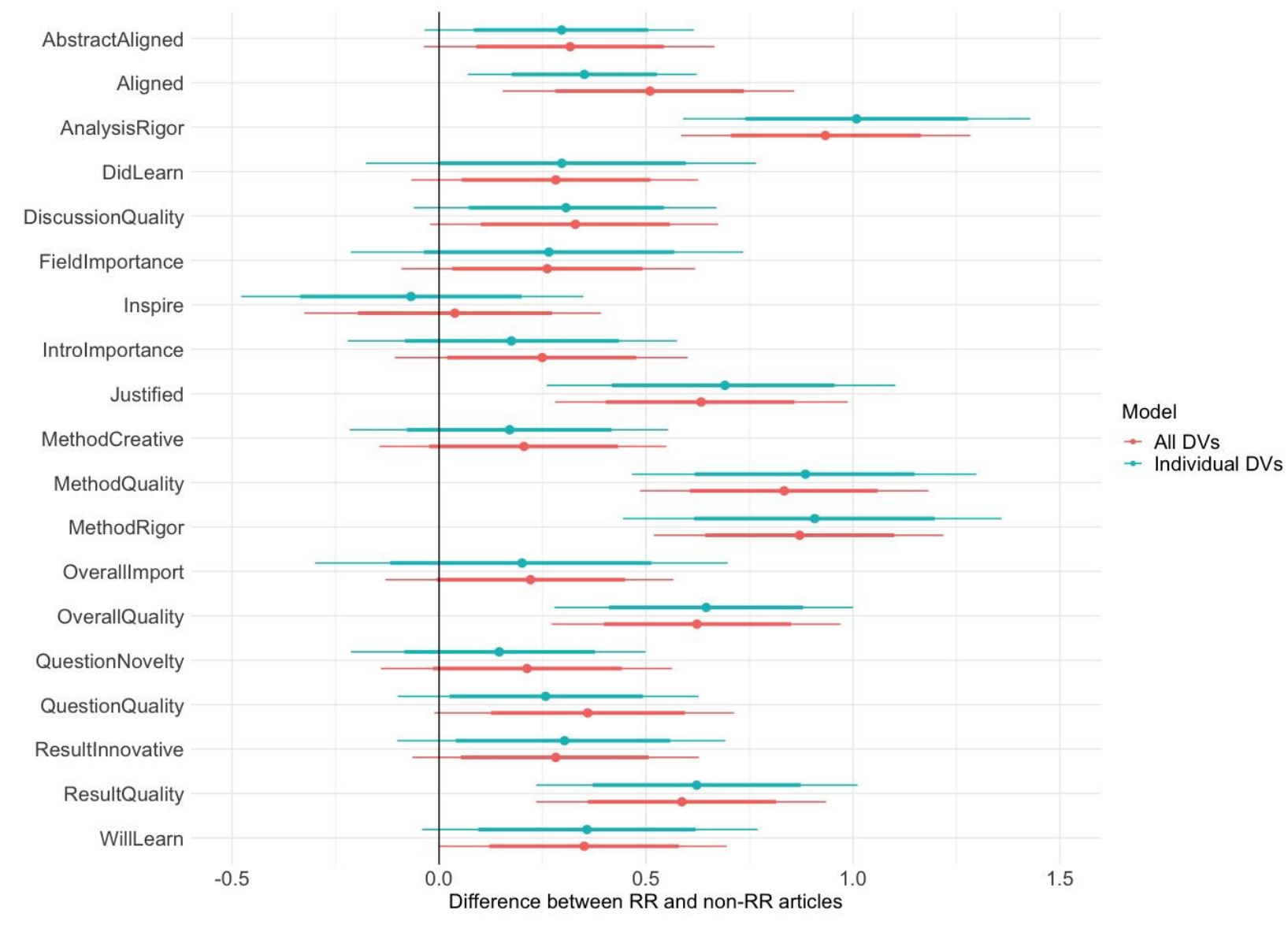




\section{Supplementary Tables}

\section{Supplementary Table 1}

Posterior means and $95 \%$ credible intervals for each outcome from the between subjects model in Table S9.

\begin{tabular}{lcc}
\hline DV & Posterior Mean & $95 \%$ Crl \\
\hline AbstractAligned & 0.32 & {$[-0.04,0.67]$} \\
Aligned & 0.51 & {$[0.15,0.86]$} \\
AnalysisRigor & 0.93 & {$[0.58,1.28]$} \\
DidLearn & 0.28 & {$[-0.07,0.63]$} \\
DiscussionQuality & 0.33 & {$[-0.02,0.67]$} \\
Fieldlmportance & 0.26 & {$[-0.09,0.62]$} \\
Inspire & 0.04 & {$[-0.33,0.39]$} \\
Introlmportance & 0.25 & {$[-0.11,0.60]$} \\
Justified & 0.63 & {$[0.28,0.99]$} \\
MethodCreative & 0.21 & {$[-0.14,0.55]$} \\
MethodQuality & 0.83 & {$[0.49,1.18]$} \\
MethodRigor & 0.87 & {$[0.52,1.22]$} \\
OverallImport & 0.22 & {$[-0.13,0.57]$} \\
OverallQuality & 0.62 & {$[0.27,0.97]$} \\
QuestionNovelty & 0.21 & {$[-0.14,0.56]$} \\
QuestionQuality & 0.36 & {$[-0.01,0.71]$} \\
ResultInnovative & 0.28 & {$[-0.06,0.63]$} \\
ResultQuality & 0.59 & {$[0.23,0.93]$} \\
WillLearn & 0.35 & {$[0.00,0.69]$} \\
\hline
\end{tabular}




\section{Supplementary Table 2}

Full model results for the exploratory analysis of how respondents' familiarity with preregistration/RRs relate to the performance advantage of $\mathrm{RR}$ versus comparison articles. Brms suggested using LOO instead of WAIC for the model because $1.2 \%$ of $p$ waic estimates were greater than 0.40 . LOO estimate was 24175.9 with a standard error of 147.1 , and all Pareto K estimates were good $(\mathrm{k}<.05)$. Field, keyword_batch_comp, Order, and Match are deviation coded, so the intercept represents the difference between RR and comparisons articles across all categories.

\begin{tabular}{lccc}
\hline & Estimate & SE & 95\% CrI \\
\hline Fixed Effects & & & \\
\hline Intercept & 0.45 & 0.23 & {$[0.00,0.89]$} \\
Field1 & -0.04 & 0.19 & {$[-0.42,0.34]$} \\
Field2 & 0.06 & 0.25 & {$[-0.44,0.55]$} \\
keyword_batch_comp1 & 0.04 & 0.09 & {$[-0.15,0.21]$} \\
Order1 & -0.26 & 0.09 & {$[-0.43,-0.09]$} \\
Match1 & -0.18 & 0.08 & {$[-0.35,-0.02]$} \\
Order1:Match1 & -0.05 & 0.08 & {$[-0.21,0.10]$} \\
\hline Random Effects & & & {$[0.01,0.78]$} \\
\hline sd(Intercept): Familiarity & 0.20 & 0.21 & {$[1.35,1.59]$} \\
sd(Intercept): Participant_id & 1.46 & 0.06 & {$[0.19,0.40]$} \\
sd(Intercept): Question & 0.28 & 0.05 & {$[0.33,0.89]$} \\
sd(Intercept): RR & 0.57 & 0.14 & {$[1.42,1.47]$} \\
sigma & 1.45 & 0.01 & \\
\hline
\end{tabular}




\section{Supplementary Table 3}

Full model results for the exploratory analysis of how respondents' beliefs about the extent to which RR improve the quality and rigor of research relate to the performance advantage of RR versus comparison articles. Brms suggested using LOO instead of WAIC for the model because $1.2 \%$ of $p \_$waic estimates were greater than 0.40 . LOO estimate was 24174.6 with a standard error of 147, and all Pareto K estimates were good $(\mathrm{k}<.05)$. Field, keyword_batch_comp, Order, and Match are deviation coded, so the intercept represents the difference between RR and comparisons articles across all categories.

\begin{tabular}{lccc}
\hline & Estimate & SE & 95\% Crl \\
\hline Fixed Effects & & & \\
\hline Intercept & 0.46 & 0.25 & {$[-0.03,0.95]$} \\
Field1 & -0.08 & 0.19 & {$[-0.46,0.31]$} \\
Field2 & 0.09 & 0.25 & {$[-0.40,0.57]$} \\
keyword_batch_comp1 & 0.03 & 0.09 & {$[-0.15,0.22]$} \\
Order1 & -0.26 & 0.08 & {$[-0.42,-0.10]$} \\
Match1 & -0.20 & 0.08 & {$[-0.36,-0.03]$} \\
Order1:Match1 & -0.06 & 0.08 & {$[-0.21,0.10]$} \\
\hline Random Effects & & & {$[0.03,0.96]$} \\
\hline sd(Intercept): Improve & 0.36 & 0.24 & {$[1.34,1.58]$} \\
sd(Intercept): Participant_id & 1.45 & 0.06 & {$[0.19,0.41]$} \\
sd(Intercept): Question & 0.28 & 0.05 & {$[0.33,0.88]$} \\
sd(Intercept): RR & 0.57 & 0.14 & {$[1.42,1.47]$} \\
sigma & 1.45 & 0.01 & \\
\hline
\end{tabular}




\section{Supplementary Table 4}

Full model results for the exploratory analysis of how respondents ability to guess whether the article they read were $R R$ or not related to the performance advantage of $R R$ versus comparison articles. Brms suggested using LOO instead of WAIC for the model because $1.4 \%$ of p_waic estimates were greater than 0.40 . LOO estimate was 24149.9 with a standard error of 147.8, and all Pareto K estimates were good ( $k$ < .05). Field, keyword_batch_comp, Order, Match are deviation coded and guessed_right is dummy coded with 'none' as the reference category. The intercept represents the difference between RR and comparisons articles for those who guessed neither article type correctly, across all other categories.

\begin{tabular}{lccc}
\hline & Estimate & SE & 95\% CrI \\
\hline Fixed Effects & & & \\
\hline Intercept & 0.10 & 0.23 & {$[-0.37,0.55]$} \\
Field1 & -0.03 & 0.20 & {$[-0.42,0.36]$} \\
Field2 & 0.06 & 0.26 & {$[-0.45,0.59]$} \\
keyword_batch_comp1 & 0.05 & 0.09 & {$[-0.13,0.23]$} \\
guessed_right2 & 0.44 & 0.22 & {$[0.02,0.87]$} \\
guessed_right3 & 0.49 & 0.24 & {$[0.00,0.95]$} \\
Order1 & -0.23 & 0.09 & {$[-0.40,-0.06]$} \\
Match1 & -0.17 & 0.08 & {$[-0.33,-0.01]$} \\
Order1:Match1 & -0.04 & 0.08 & {$[-0.20,0.13]$} \\
\hline Random Effects & & & \\
\hline sd(Intercept): RR & 0.58 & 0.14 & {$[0.33,0.90]$} \\
sd(Intercept): question & 0.15 & 0.05 & {$[0.05,0.27]$} \\
sd(guessed_right2): question & 0.17 & 0.07 & {$[0.05,0.31]$} \\
sd(guessed_right3): question & 0.30 & 0.08 & {$[0.17,0.48]$} \\
cor(Intercept,guessed_right2): question & 0.33 & 0.36 & {$[-0.42,0.92]$} \\
cor(Intercept,guessed_right3): question & 0.64 & 0.25 & {$[0.03,0.97]$} \\
cor(guessed_right2,guessed_right3): question & 0.72 & 0.23 & {$[0.13,0.98]$} \\
sd(Intercept): participant_id & 1.46 & 0.06 & {$[1.34,1.58]$} \\
sigma & & 0.01 & {$[1.42,1.47]$} \\
\hline
\end{tabular}




\section{Supplementary Table 5}

Registered Reports and comparison articles digital object identifiers (DOIs).

\begin{tabular}{|c|c|c|c|}
\hline RR pair & Registered Report & $\begin{array}{l}\text { Author-based } \\
\text { comparison }\end{array}$ & $\begin{array}{c}\text { Journal-based } \\
\text { comparison }\end{array}$ \\
\hline RR01 & $10.3758 / s 13414-017-1304-y$ & $10.3758 / \mathrm{s} 13423-015-0941-1$ & $10.3758 / \mathrm{s} 13414-016-1221-5$ \\
\hline RR05 & $10.1080 / 23743603.2016 .1273647$ & $10.1177 / 0146167217727496$ & 10.1027/1864-9335/a000325 \\
\hline RR06 & $10.1080 / 23743603.2017 .1288877$ & $10.1007 / \mathrm{s} 10919-017-0256-1$ & 10.1027/1864-9335/a000275 \\
\hline RR07 & $10.1080 / 23743603.2017 .1358477$ & 10.1016/j.jesp.2016.12.009 & 10.1027/1864-9335/a000298 \\
\hline RR08 & $10.1080 / 23743603.2017 .1360573$ & 10.1002/ejsp.2321 & $10.1027 / 1864-9335 / a 000312$ \\
\hline RR09 & $10.1080 / 23743603.2017 .1376580$ & 10.1037/хар0000153 & $10.1027 / 1864-9335 / a 000317$ \\
\hline RR10 & $10.1080 / 23743603.2017 .1404427$ & $10.1177 / 0093650217699935$ & $10.1027 / 1864-9335 / a 000316$ \\
\hline RR11 & $10.1080 / 23743603.2018 .1425089$ & $10.1037 / p s p l 0000087$ & $10.1027 / 1864-9335 / a 000313$ \\
\hline RR12 & $10.1080 / 23743603.2018 .1436939$ & 10.1016/j.jrp.2017.11.003 & $10.1027 / 1864-9335 / a 000320$ \\
\hline RR13 & 10.1016/j.cortex.2014.12.019 & 10.1016/j.brainres.2014.11.046 & 10.1016/j.cortex.2015.06.027 \\
\hline RR14 & 10.1016/j.cortex.2015.03.010 & 10.1162/jocn_a_00885 & 10.1016/j.cortex.2014.08.026 \\
\hline RR15 & 10.1016/j.cortex.2016.01.006 & 10.1016/j.cortex.2015.03.004 & 10.1016/j.cortex.2014.11.014 \\
\hline RR16 & 10.1016/j.cortex.2016.03.004 & 10.1523/jneurosci.0903-15.2015 & 10.1016/j.cortex.2016.03.005 \\
\hline RR17 & 10.1016/j.cortex.2016.03.019 & 10.1038/ejhg.2015.168 & 10.1016/j.cortex.2016.04.015 \\
\hline RR18 & 10.1016/j.cortex.2017.09.026 & 10.1111/psyp.12732 & 10.1016/j.cortex.2017.09.001 \\
\hline RR19 & 10.1016/j.cortex.2018.02.009 & 10.1093/cercor/bhw262 & 10.1016/j.cortex.2017.06.022 \\
\hline RR20 & 10.1016/j.drugalcdep.2017.08.022 & 10.1016/j.cogpsych.2017.02.002 & 10.1016/j.drugalcdep.2016.11.016 \\
\hline RR21 & $10.1027 / 1618-3169 / a 000364$ & $10.1080 / 13546783.2017 .1374306$ & 10.1027/1618-3169/a000379 \\
\hline RR22 & 10.3389/fpsyg.2015.00672 & $10.2139 /$ ssrn.2351377 & 10.3389/fpsyg.2015.01553 \\
\hline RR31 & 10.1027/1864-1105/a000208 & $10.3758 / \mathrm{s} 13423-017-1322-8$ & $10.1027 / 1864-1105 / a 000174$ \\
\hline RR32 & $10.1027 / 1864-1105 / a 000211$ & 10.1016/j.chb.2015.08.048 & $10.1027 / 1864-1105 / a 000168$ \\
\hline RR33 & $10.1027 / 1864-1105 / a 000210$ & NA & $10.1027 / 1864-1105 / a 000177$ \\
\hline RR34 & 10.1027/1864-1105/a000209 & $10.5817 / \mathrm{cp} 2016-4-3$ & $10.1027 / 1864-1105 / a 000215$ \\
\hline RR35 & 10.1016/j.jrp.2017.10.005 & 10.1111/jopy.12183 & 10.1016/j.jrp.2017.05.003 \\
\hline RR36 & 10.1016/j.jrp.2017.12.003 & $10.3758 / \mathrm{s} 13428-015-0646-4$ & 10.1016/j.jrp.2015.11.002 \\
\hline RR37 & 10.1016/j.jrp.2018.01.002 & 10.1016/j.comppsych.2016.10.003 & 10.1016/j.jrp.2016.10.002 \\
\hline RR39 & 10.1098/rsos. 160935 & 10.1521/soco.2016.34.5.382 & $10.1098 /$ rsos. 170270 \\
\hline RR40 & 10.1098/rsos. 171678 & 10.1016/j.lindif.2017.04.001 & $10.1098 /$ rsos. 170121 \\
\hline RR41 & $10.1098 /$ rsos. 170543 & 10.1016/j.evolhumbehav.2017.08.001 & $10.1098 /$ rsos. 171268 \\
\hline
\end{tabular}




\section{Supplementary Table 6}

The number of participants who completed at least one question for both the RR and control article for each RR article pair. Across all RR pairs, 353 participants answered at least one outcome on the first paper they reviewed. Shown here are the 351 participants who completed at least one pair of questions from both papers (i.e., 2 participants dropped out before completing the second review).

\begin{tabular}{|c|c|}
\hline RR Pair & $\mathbf{N}$ \\
\hline RR40 & 24 \\
\hline RR36 & 23 \\
\hline RR09 & 21 \\
\hline RR20 & 21 \\
\hline RR01 & 19 \\
\hline RR19 & 18 \\
\hline RR07 & 16 \\
\hline RR34 & 15 \\
\hline RR11 & 14 \\
\hline RR41 & 14 \\
\hline RR10 & 13 \\
\hline RR37 & 13 \\
\hline RR39 & 13 \\
\hline RR05 & 12 \\
\hline RR08 & 12 \\
\hline RR35 & 12 \\
\hline RR15 & 11 \\
\hline RR14 & 10 \\
\hline RR21 & 10 \\
\hline RR16 & 9 \\
\hline RR32 & 9 \\
\hline RR06 & 8 \\
\hline RR12 & 8 \\
\hline RR17 & 8 \\
\hline RR18 & 6 \\
\hline RR22 & 5 \\
\hline RR31 & 4 \\
\hline RR33 & 3 \\
\hline
\end{tabular}




\section{Supplementary Table 7}

Model results for the within-subjects model in which all DVs are included through a multilevel model with partial-pooling. We implemented aAll analyses were implemented using the $R$ package brms. ${ }^{44}$ Brms suggested using LOO instead of WAIC for the model because $1.2 \%$ of p_waic estimates were greater than 0.40 . LOO estimate was 24175.8 with a standard error of 147 , and all Pareto K estimates were good $(\mathrm{k}<.05)$. Field, keyword_batch_comp, Order, and Match are deviation coded, so the intercept represents the difference between RR and comparisons articles across all categories.

\begin{tabular}{lccc}
\hline & Estimate & SE & $95 \%$ Crl \\
\hline Fixed Effects & & & \\
\hline Intercept & 0.46 & 0.18 & {$[0.10,0.82]$} \\
Field1 & -0.04 & 0.19 & {$[-0.43,0.34]$} \\
Field2 & 0.07 & 0.26 & {$[-0.44,0.60]$} \\
keyword_batch_comp1 & 0.03 & 0.09 & {$[-0.15,0.22]$} \\
Order1 & -0.26 & 0.08 & {$[-0.42,-0.11]$} \\
Match1 & -0.18 & 0.08 & {$[-0.34,-0.01]$} \\
Order1:Match1 & -0.05 & 0.08 & {$[-0.22,0.10]$} \\
\hline Random Effects & & & {$[1.36,1.59]$} \\
\hline sd(Intercept): Participant_id & 1.46 & 0.06 & {$[0.19,0.40]$} \\
sd(Intercept): Question & 0.28 & 0.05 & {$[0.33,0.90]$} \\
sd(Intercept): RR & 0.58 & 0.15 & {$[1.42,1.47]$} \\
sigma & 1.45 & 0.01 & \\
\hline
\end{tabular}




\section{Supplementary Table 8}

Model results for the between-subjects model in which all DVs are included through a multilevel model with partial-pooling. Brms suggested using LOO instead of WAIC for the model because $1.4 \%$ of $p_{-}$waic estimates were greater than 0.40 . LOO estimate was 24149.9 with a standard error of 147.8, and all Pareto K estimates were good $(k<.05)$. Field, keyword_batch_comp, and Match are deviation coded and article_type is dummy coded with 'alternative' as the reference category. Article_type represents the performance difference between RR and comparison articles across all other categories.

\begin{tabular}{lccc}
\hline & Estimate & SE & $95 \%$ Crl \\
\hline Fixed Effects & & & \\
\hline Intercept & -0.04 & 0.12 & {$[-0.28,0.21]$} \\
Field1 & 0.00 & 0.11 & {$[-0.22,0.21]$} \\
Field2 & 0.04 & 0.14 & {$[-0.23,0.31]$} \\
keyword_batch_comp1 & -0.05 & 0.07 & {$[-0.19,0.09]$} \\
article_type2 & 0.43 & 0.16 & {$[0.10,0.74]$} \\
Match1 & 0.04 & 0.09 & {$[-0.14,0.21]$} \\
article_type2:Match1 & -0.21 & 0.13 & {$[-0.46,0.04]$} \\
\hline Random Effects & & & \\
\hline sd(Intercept): RR & 0.21 & 0.13 & {$[0.01,0.49]$} \\
sd(article_type2): RR & 0.30 & 0.18 & {$[0.02,0.69]$} \\
cor(Intercept,article_type2): RR & -0.32 & 0.53 & {$[-0.97,0.87]$} \\
sd(Intercept): question & 0.22 & 0.05 & {$[0.15,0.33]$} \\
sd(article_type2): question & 0.30 & 0.07 & {$[0.20,0.45]$} \\
cor(Intercept,article_type2): question & 0.32 & 0.25 & {$[-0.21,0.75]$} \\
sd(Intercept): participant_id & 1.16 & 0.05 & {$[1.07,1.26]$} \\
sigma & 1.10 & 0.01 & {$[1.08,1.12]$} \\
\hline
\end{tabular}




\section{Supplementary Table 9}

Descriptive statistics of the all studies of Registered Reports and comparison articles separated by author-based and journal-based

\begin{tabular}{|c|c|c|c|}
\hline & $\begin{array}{c}\text { Registered } \\
\text { Report }\end{array}$ & $\begin{array}{l}\text { Author-based } \\
\text { comparison }\end{array}$ & $\begin{array}{c}\text { Journal-based } \\
\text { comparison }\end{array}$ \\
\hline$N$ & 29 & 28 & 29 \\
\hline \multicolumn{4}{|c|}{ Statcheck results (all studies) } \\
\hline Detectable statistical tests & $15(52 \%)$ & $11(39 \%)$ & $12(41 \%)$ \\
\hline \multicolumn{4}{|l|}{ Errors } \\
\hline$M d$ & 0 & 0 & 0 \\
\hline$M(S D)$ & $0.87(1.13)$ & $0.55(0.69)$ & $0.75(1.22)$ \\
\hline Papers with $>0$ errors & 6 & 5 & 4 \\
\hline \multicolumn{4}{|c|}{ Statistical significance decision errors } \\
\hline$M d$ & 0 & 0 & 0 \\
\hline$M(S D)$ & $0.07(0.26)$ & $0.09(0.30)$ & $0.25(0.62)$ \\
\hline Papers with $>0$ errors & 1 & 1 & 2 \\
\hline \multicolumn{4}{|c|}{$\begin{array}{l}\text { Notes: Statcheck "papers with detectable statistical tests" includes papers for which statcheck } \\
\text { detected at least one statistical result, namely correlations and } t, F, X 2, Z \text {, and } Q \text { tests, reported } \\
\text { in APA style; "errors" indicates all detected statistical reporting errors; "decision errors" indicates } \\
\text { results reported as statistically significant when recomputed results were not, or vice versa. In } \\
\text { principle, all Registered Reports were preregistered with the journal, but papers were not } \\
\text { counted if a preregistration could not be found in an independent registry. }\end{array}$} \\
\hline
\end{tabular}




\section{Supplementary Table 10}

Article keywords included in the survey sample.

\begin{tabular}{|c|c|c|}
\hline RR pair & Discipline & Article keywords \\
\hline RR01 & Cognitive Psychology & $\begin{array}{l}\text { Response time model, Predictions, Intelligence, Sequential } \\
\text { sampling models, Risky choice, Value based decisions, } \\
\text { Theoretical and computational models, Reaction time } \\
\text { methods, Spatial vision }\end{array}$ \\
\hline RR05 & $\begin{array}{l}\text { Social-Personality } \\
\text { Psychology (Positive } \\
\text { Psychology) }\end{array}$ & $\begin{array}{l}\text { Prosocial behavior, Education, Well-being, College } \\
\text { students, Bayesian statistics, Social comparison, } \\
\text { Interpersonal relationships, Person-environment fit, Dignity } \\
\text { self-construal, Sense of belonging to the university, } \\
\text { Academic success }\end{array}$ \\
\hline RR06 & $\begin{array}{l}\text { Social-Personality } \\
\text { Psychology (Attitudes } \\
\text { and Social Cognition) }\end{array}$ & $\begin{array}{l}\text { Construal-level theory, Psychological distance, Goal } \\
\text { contagion, Action Identification, Motivation, Embodiment, } \\
\text { Gestures, Communication, Social dilemma, Public good } \\
\text { dilemma, Self-interest, Fairness, Group goal, Allocation } \\
\text { preference }\end{array}$ \\
\hline RR07 & $\begin{array}{l}\text { Social-Personality } \\
\text { Psychology (Group } \\
\text { Processes and Intergro } \\
\text { Relations) }\end{array}$ & $\begin{array}{l}\text { Ostracism, Intergroup attribution, Ultimate attribution error, } \\
\text { Subjective group dynamics, Black sheep effect, Police } \\
\text { officer's dilemma, Shooter bias, Avoidance bias, } \\
\text { Stereotypes, Threat, Replication, Reactive/proactive } \\
\text { aggression, Social rejection, Aggressive peers, Academic } \\
\text { failure, Childhood, Adolescence, Germany }\end{array}$ \\
\hline RR08 & $\begin{array}{l}\text { Social-Personality } \\
\text { Psychology (Group } \\
\text { Processes and Intergro } \\
\text { Relations) }\end{array}$ & $\begin{array}{l}\text { Schadenfreude, Asylum seekers, National identification, } \\
\text { Cross-cultural, Threat, Blame, Perceived } \\
\text { o perspective-taking, Bullying, Inferred moral emotions, } \\
\text { Trust, Forgiveness, Severity of harm, Refugees, } \\
\text { Stereotypes, Labels, Language, Social perception }\end{array}$ \\
\hline RR09 & $\begin{array}{l}\text { Social-Personality } \\
\text { Psychology (Positive } \\
\text { Psychology) }\end{array}$ & $\begin{array}{l}\text { Happiness, Meaning, Subjective well-being, Hedonia, } \\
\text { Eudaimonia, Future self-continuity, Exercise, Health, } \\
\text { Boredom, Impulsiveness, Meaning, Self-awareness, } \\
\text { Existential psychology }\end{array}$ \\
\hline RR10 & $\begin{array}{l}\text { Social-Personality } \\
\text { Psychology (Attitudes } \\
\text { and Social Cognition) }\end{array}$ & $\begin{array}{l}\text { Processing fluency, Language complexity, Policy attitudes, } \\
\text { Attitudes, Minority opinions, Attitude strength, } \\
\text { Knowledgeability, Informational influence, Normative } \\
\text { influence, Attitude certainty, Spatial orientation, Moral } \\
\text { judgments, Morality, Side biases, Fluency }\end{array}$ \\
\hline
\end{tabular}




\section{RR pair Discipline Article keywords}

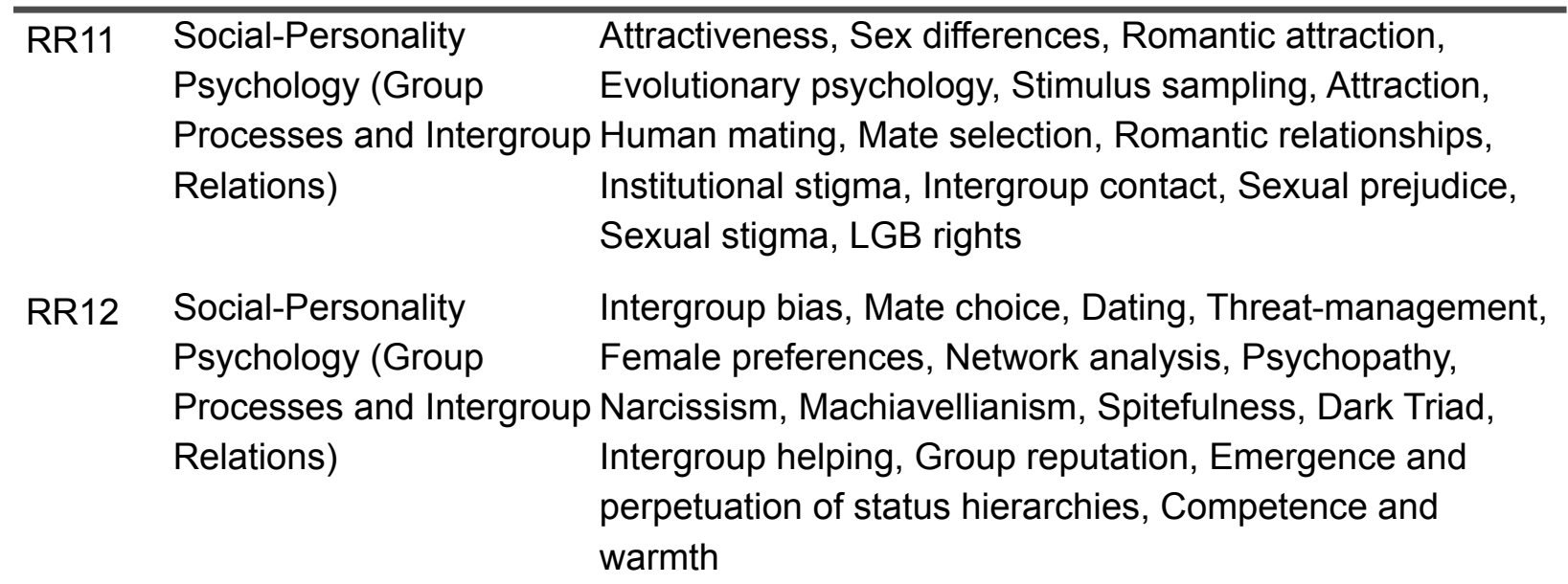

RR13 Neuroscience

P600, P3, Single-trial analysis, Sentence processing, Ventral attention network, ERPs, Animacy, Tamil, Actor, Predictive processing, Contextual cues, Sentence comprehension, Time-frequency analysis, Source localization, Theta oscillations, Verbal working memory

RR14 Neuroscience Internal model, Audiovisual speech, Context, ERP, Oscillations, Transcranial direct current stimulation, Dorsolateral prefrontal cortex, Probabilistic learning task, Exploration, Exploitation, Working memory

RR15 Neuroscience Taxonomic category, Thematic category, Features, The organization of object knowledge, Diffusion tensor imaging, Language production and comprehension, Lexical-semantic deficits, Semantic interference, Lesion-symptom mapping, Categorization, Language, Right hemisphere, TMS, Typicality

RR16 Neuroscience MEG, Visual cortex, Gamma oscillations, AMPA receptors, Perampanel, Alpha rhythms, Depression, Dynamic causal modelling, Gamma rhythms, Ketamine, Magnetoencephalography, Letters processing, Visual perception, VEP

RR17 Neuroscience

Mirror neurons, EEG, Frequency, Mu suppression, Alpha, Beta, Methods, Baseline, Mirror visual feedback, Visual perception, Motor cortex, Event related potential, Lateralized readiness potential 


\begin{tabular}{|c|c|c|}
\hline RR pair & Discipline & Article keywords \\
\hline RR18 & Neuroscience & $\begin{array}{l}\text { Exercise, Endurance, Prefrontal cortex, Motor cortex, } \\
\text { tDCS, NIRS, Intrinsic motivation, Cortical excitability, } \\
\text { Single-pulse TMS, TMS, Motor awareness, Premotor } \\
\text { cortex, Posterior parietal cortex }\end{array}$ \\
\hline RR19 & Neuroscience & $\begin{array}{l}\text { Attentional capture, Reward, Motivation, Spatial attention, } \\
\text { Brain stimulation, Vestibular stimulation, Galvanic } \\
\text { vestibular stimulation, Anterior cingulate cortex, Addiction, } \\
\text { Atomoxetine, Fronto-parietal network, Locus coeruleus, } \\
\text { Monkey, Resting-state fMRI, Attention, Short-term memory, } \\
\text { Long-term memory, CDA }\end{array}$ \\
\hline RR20 & Cognitive Psychology & $\begin{array}{l}\text { Alcohol, Inhibition, Manual, Saccade, SSRT, Action } \\
\text { selection, Modelling, Response modalities, Competition, } \\
\text { Reaction time, Response inhibition, Error processing, } \\
\text { Performance monitoring, Cognitive control }\end{array}$ \\
\hline RR21 & $\begin{array}{l}\text { Social-Personality } \\
\text { Psychology (Attitudes } \\
\text { and Social Cognition) }\end{array}$ & $\begin{array}{l}\text { Affect misattribution procedure, Implicit measures, } \\
\text { Prejudice effects, Relevance, Truth conditions, Indicative } \\
\text { conditionals, Probability, Conjunctions, Affect, Familiarity, } \\
\text { Intention, Misattribution }\end{array}$ \\
\hline RR22 & $\begin{array}{l}\text { Social-Personality } \\
\text { Psychology (Attitudes } \\
\text { and Social Cognition) }\end{array}$ & $\begin{array}{l}\text { Deception detection, Physiological markers, Skin } \\
\text { temperature, Indirect deception detection, Interpersonal } \\
\text { relations, Psychophysiology, Deception, Cognitive } \\
\text { processing capacity, Morality, Dual system, } \\
\text { Decision-making, Psychophysiology, Expectation, } \\
\text { Presentiment, Consciousness, Lie detection }\end{array}$ \\
\hline RR31 & $\begin{array}{l}\text { Social-Personality } \\
\text { Psychology } \\
\text { (Media/Applied } \\
\text { Psychology) }\end{array}$ & $\begin{array}{l}\text { Online education, Multimedia, Subtitles, Video, MOOCs, } \\
\text { Mind-wandering, Task-unrelated thoughts, Framing, } \\
\text { Response bias, Cognitive biases, Educational games, } \\
\text { Game characteristics, Character customization, Digital } \\
\text { games }\end{array}$ \\
\hline RR32 & $\begin{array}{l}\text { Social-Personality } \\
\text { Psychology (Positive } \\
\text { Psychology \& } \\
\text { Media/Applied } \\
\text { Psychology) }\end{array}$ & $\begin{array}{l}\text { Prosocial behavior, Interactive narrative, Appreciation, } \\
\text { Games for change, Yoked design, Gamification, Motivation, } \\
\text { Self-determination theory, Video games, Interactivity, FAE, } \\
\text { Human-computer interaction, Identification }\end{array}$ \\
\hline
\end{tabular}




\begin{tabular}{|c|c|c|}
\hline RR pair & Discipline & Article keywords \\
\hline RR33 & $\begin{array}{l}\text { Social-Personality } \\
\text { Psychology } \\
\text { (Media/Applied } \\
\text { Psychology) }\end{array}$ & $\begin{array}{l}\text { Video games, Risk, College, University health, Video game } \\
\text { addiction, Online gaming, Excessive game use, Transient } \\
\text { phenomenon, Longitudinal survey, Pathological gaming }\end{array}$ \\
\hline RR34 & $\begin{array}{l}\text { Social-Personality } \\
\text { Psychology (Positive } \\
\text { Psychology \& } \\
\text { Media/Applied } \\
\text { Psychology) }\end{array}$ & $\begin{array}{l}\text { Video games, Social play, Reciprocity, Prosocial behavior, } \\
\text { Challenge, Social capital, Informational benefits, Social } \\
\text { media, Ambient awareness, Longitudinal, Social network, } \\
\text { Social network sites, Video game play, Moral reasoning, } \\
\text { Cognitive load, Rational vs. intuitive processing }\end{array}$ \\
\hline RR35 & $\begin{array}{l}\text { Social-Personality } \\
\text { Psychology (Personality } \\
\text { Psychology) }\end{array}$ & $\begin{array}{l}\text { Neuroticism, Vigilance, Health, Health behaviors, Self-rated } \\
\text { health, Cross-sectional, Extraversion, Conscientiousness, } \\
\text { Interaction, Internalizing disorders, Distress disorders, } \\
\text { Depression, Trait affect, Disinhibition }\end{array}$ \\
\hline RR36 & $\begin{array}{l}\text { Social-Personality } \\
\text { Psychology (Personality } \\
\text { Psychology) }\end{array}$ & $\begin{array}{l}\text { Negotiation, Emotional intelligence, Emotion recognition } \\
\text { ability, General mental ability, Social interaction, } \\
\text { Assessment, Testing, Factor analysis, Behavioral } \\
\text { problems, Effortful control, Emotion, Academic } \\
\text { engagement, Student-teacher relationships, Temperament }\end{array}$ \\
\hline RR37 & $\begin{array}{l}\text { Social-Personality } \\
\text { Psychology (Personality } \\
\text { Psychology) }\end{array}$ & $\begin{array}{l}\text { Childhood environments, Defense mechanisms, } \\
\text { Nurturance, Midlife functioning, Adaptive defenses, } \\
\text { Personality development, Adversity, Life events, Stress, Big } \\
\text { Five traits, Personality change, Neuroticism, } \\
\text { Conscientiousness, Agreeableness, Openness }\end{array}$ \\
\hline RR39 & Cognitive Psychology & $\begin{array}{l}\text { Evaluative conditioning, Implicit learning, Cross-modal } \\
\text { conditioning, Awareness, Incidental learning, Generative } \\
\text { models, Probability learning }\end{array}$ \\
\hline RR40 & Cognitive Psychology & $\begin{array}{l}\text { Statistical learning, Verbal working memory, Incidental } \\
\text { learning, Online learning, Non-adjacent dependencies, } \\
\text { Probabilistic dependencies, Genetics, Speech, Word } \\
\text { reading, Chinese, Observational learning, Social } \\
\text { facilitation, Spatial memory, Rat }\end{array}$ \\
\hline RR41 & $\begin{array}{l}\text { Social-Personality } \\
\text { Psychology (Attitudes } \\
\text { and Social Cognition) }\end{array}$ & $\begin{array}{l}\text { Behavioural economics, Ultimatum game, Cultural } \\
\text { evolution, Evolutionary psychology, Conformity, } \\
\text { Drift-diffusion models, Social information, Norms, Social } \\
\text { influence, Decision-making }\end{array}$ \\
\hline
\end{tabular}




\section{Supplementary References}

40. Ellard-Gray, A., Jeffrey, N. K., Choubak, M. \& Crann, S. E. Finding the Hidden Participant: Solutions for Recruiting Hidden, Hard-to-Reach, and Vulnerable Populations. Int. J. Qual. Methods (2015) doi:10.1177/1609406915621420.

41. R Core Team. R: A language and environment for statistical computing. (2019).

42. Blaette, A. \& Leonhardt, C. trickypdf: Turn tricky pdf into txt/xml for corpus preparation. $R$ package version 0.1.2. (2019).

43. Wickham, H., Hester, J. \& Ooms, J. xm/2.R package version 1.2.5. (2020).

44. Bürkner, P.-C. brms: An R Package for Bayesian Multilevel Models Using Stan. J. Stat. Softw. 80, 1-28 (2017). 